\title{
Colloid Characterization and
}

Quantification in Groundwater Samples

K. Stephen Kung

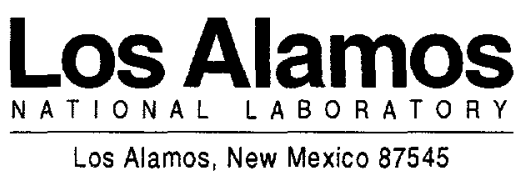





\section{DISCLAIMER}

This report was prepared as an account of work sponsored by an agency of the United States Government. Neither the United States Government nor any agency thereof, nor any of their employees, make any warranty, express or implied, or assumes any legal liability or responsibility for the accuracy, completeness, or usefulness of any information, apparatus, product, or process disclosed, or represents that its use would not infringe privately owned rights. Reference herein to any specific commercial product, process, or service by trade name, trademark, manufacturer, or otherwise does not necessarily constitute or imply its endorsement, recommendation, or favoring by the United States Government or any agency thereof. The views and opinions of authors expressed herein do not necessarily state or reflect those of the United States Government or any agency thereof. 


\section{DISCLAIMER}

Portions of this document may be illegible in electronic image products. Images are produced from the best available original document. 


\section{Table of Contents}

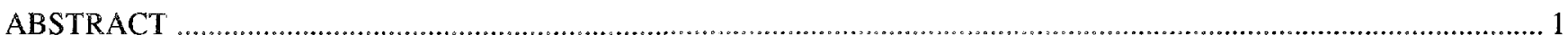

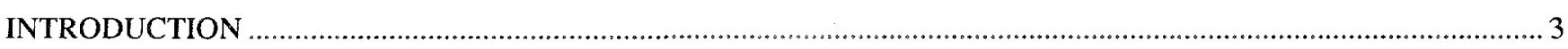

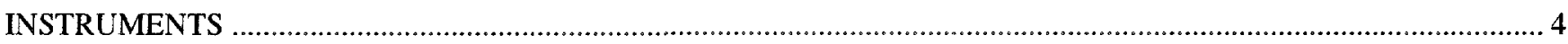

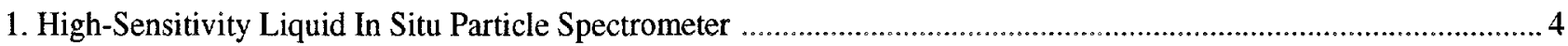

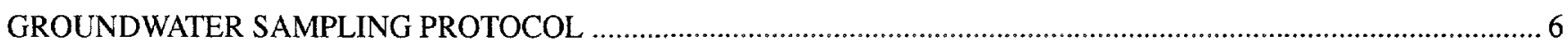

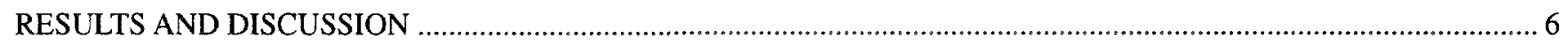

Task I. Colloid Size Distribution and Concentration Analyses for ER-20-5-1 Samples ...................................................6 6

Task II. Effects of the Sample Container, Storage, and Aging Time on Colloid Stability ......................................... 14

Task III. Colloid Characterization of Groundwater Collected from ER-20-5, \#1 and \#3 Wells .................................... 22

Task IV. Colloid Analyses of Cheshire, U20n, Groundwater Samples ............................................................... 33 


\section{List of Tables}

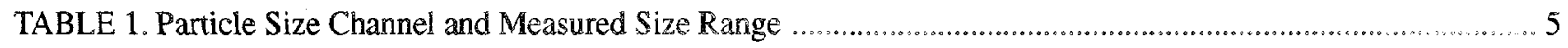

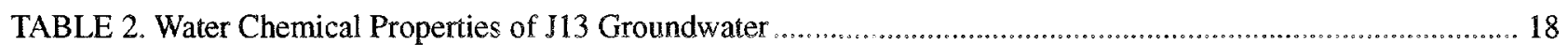

\section{List of Figures}

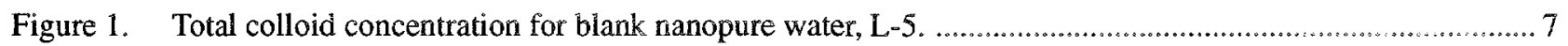

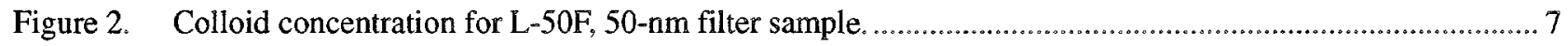

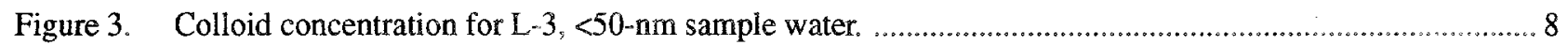

Figure 4. Colloid concentration for $\mathrm{L}-6 \mathrm{~F} ; 100,000$-nmwl filter water. ........................................................... 8

Figure 5. Colloid concentration L-4, $<100,000$-nmwl sample water. .............................................................. 9

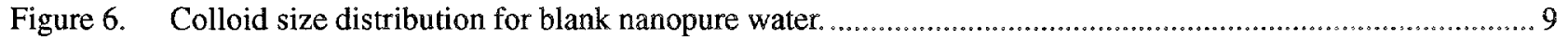

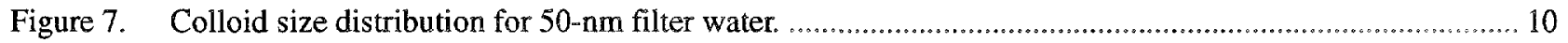

Figure 8. Colloid size distribution for $<50-\mathrm{nm}$ nmwl filter water .................................................................. 10

Figure 9. Colloid size distribution for $100,000-n m w l$ filter water. ............................................................. 11

Figure 10. Colloid size distribution for $<100,000$-nmwl filter sample. ......................................................... 11

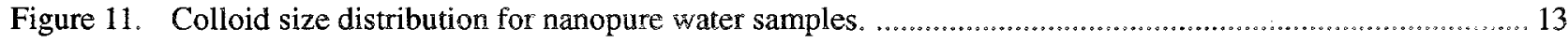

Figure 12. Total colloid concentrations for nanopure water samples. ............................................................ 13

Figure 13. Colloid size distribution for $\mathrm{J} 13$ water stored in different types of containers. ................................... 15

Figure 14. Total colloid concentration between the sizes of 50 and $200 \mathrm{~nm}$ in J13 water samples with and without filtrations.

Figure 15. Colloid size distribution for J13 water stored in a 200-liter barrel and filtered through 0.2 - and $0.45-\mu \mathrm{m}$ nylon filters.

Figure 16. Colloid size distribution for unfiltered J13 water stored in Teflon bottles before and after being degassed.

Figure 17. Colloid size distribution for $0.2-\mu \mathrm{m}$ filtered $\mathrm{J} 13$ water stored in a Teflon bottle before and after being degassed.

Figure 18. Colloid size distribution for $0.45-\mu \mathrm{m}$ filtered J13 water stored in a Teflon bottle before and after being degassed.

Figure 19. Colloid concentrations in filtered and unfiltered J13 barrel samples after degassing in a Teflon bottle.... 19

Figure 20. Colloid size distribution for unfiltered J13 water before and after being stored in a Teflon bottle for 190 days. 
Figure 21. Colloid size distribution for unfiltered $\mathbf{J} 13$ water before and after being stored in a Teflon bottle for 190 days.

Figure 22. Colloid size distribution for $0.2-\mu \mathrm{m}$ filtered $\mathrm{J} 13$ water after being stored in a Teflon bottle for 190 days.

Figure 23. Colloid size distribution for $0.45-\mu \mathrm{m}$ filtered $\mathrm{J} 13$ water before and after being stored in a Teflon bottle for 190 days.

Figure 24. Colloid size distribution for unfiltered J13 water after being stored for 190 days.

Figure 25. Total colloid concentration for $\mathrm{J} 13$ water before and after being stored for 190 days. .22

Figure 26. Total colloid concentration for the ER-20-5 Well \#1.

Figure 27. Colloid size distribution for the ER-20-5 Well \#1 water.

Figure 28. EDS results collected from the scanning electron microscope of colloids in ER-20-5 Well \#1 groundwater. 24

Figure 29. EDS results obtained from the TEM of colloidal material in ER-20-5 Well \#1 groundwater. .25

Figure 30. A transmission electron micrograph of colloidal particles collected from ER-20-5

Well \#1 groundwater.

Figure 31. A transmission electron micrograph of colloidal particles collected from ER-20-5

Well \#1 groundwater.

Figure 32. A transmission electron micrograph of colloidal particles collected from ER-20-5

Well \#1 groundwater.

Figure 33. High-resolution transmission electron micrograph of colloidal particles collected from ER-20-5

Well \#1 groundwater.

Figure 34. The transmission electron micrograph shows lattice fringe images of colloidal material collected from

ER-20-5 Well \#1 groundwater.

Figure 35. The high-resolution transmission electron micrograph shows lattice fringe images and computergenerated diffraction patterns of colloidal material collected from ER-20-5 Well \#1 groundwater. 31

Figure 36. Colloid size distribution for the ER-20-5 Wells \#1 and \#3 samples. 32

Figure 37. Total colloid concentration for ER-20-5 Wells \#1 and \#3.

Figure 38. Unfiltered and $0.45-\mu \mathrm{m}$ filtered colloid size distribution in groundwater samples collected from

Cheshire, U-20n.

Figure 39. Unfiltered and $0.45-\mu \mathrm{m}$ filtered colloid concentration in groundwater samples collected from Cheshire, U-20n. 34

Figure 40. Colloid size distribution in groundwater samples collected from Cheshire, U-20n. 35

Figure 41. Total colloid concentration in groundwater samples collected from Cheshire, U-20n. 36 
a

-

0

- 
COLLOID CHARACTERIZATION AND

QUANTIFICATION IN GROUNDWATER SAMPLES

by

K. Stephen Kung

\begin{abstract}
This report describes the work conducted at Los Alamos National Laboratory for studying the groundwater colloids for the Yucca Mountain Project in conjunction with the Hydrologic Resources Management Program (HRMP) and the Underground Test Area (UGTA) Project. Colloidal particle size distributions and total particle concentration in groundwater samples are quantified and characterized. Colloid materials from cavity waters collected near underground nuclear explosion sites by HRMP field sampling personnel at the Nevada Test Site (NTS) were quantified. Selected colloid samples were further characterized by electron microscope to evaluate the colloid shapes, elemental compositions, and mineral phases.
\end{abstract}

We have evaluated the colloid size and concentration in the natural groundwater sample that was collected from the ER-20-5 well and stored in a 50-gallon (about 200-liter) barrel for several months. This groundwater sample was studied because HRMP personnel have identified trace levels of radionuclides in the water sample. Colloid results show that even though the water sample had filtered through a series of Millipore filters, high-colloid concentrations were identified in all unfiltered and filtered samples. We had studied the samples that were diluted with distilled water and found that diluted samples contained more colloids than the undiluted ones. These results imply that colloids are probably not stable during the storage conditions. Furthermore, results demonstrate that undesired colloids have been introduced into the samples during the storage, filtration, and dilution processes.

We have evaluated possible sources of colloid contamination associated with sample collection, filtrating, storage, and analyses of natural groundwaters. The effects of container types and sample storage time on colloid size distribution and total concentration were studied to evaluate colloid stability by using $\mathrm{J} 13$ groundwater.

Our data suggest that groundwater samples should be analyzed for colloid size and concentration shortly after they have been collected. A prolonged waiting period after sampling will affect the colloid size distribution as well as colloid concentration resulting from the changes of water chemical properties.

Our data also show that sample containers, filter materials, and labware that are used for colloid analyses should be cleaned by specially treated low-colloid-containing water. Water used for sample dilution should be verified for total colloidal particle concentration. We then analyzed freshly collected groundwater from NTS wells ER-20-5\#1 and \#3. Results show that these groundwater samples have similar colloid concentrations and particle size distributions. For the particle size range between 50- and 200-nm, about ten trillion (1E10) colloidal particles per liter are present in these water samples. Most of these colloidal 
particles are less than $100 \mathrm{~nm}$ in size. For example, more than $98 \%$ of the colloids are smaller than $100 \mathrm{~mm}$ in size in the ER-20-5 \#1 sample.

Furthermore, it was found that the smaller the sizes of colloid, the higher the colloid concentration present in the water.

For another site at NTS, Cheshire, we had analyzed two zones of groundwater samples. For water samples collected from the lower water zone (near the underground detonation cavity about 3,700 feet of slanted depth from the surface), the colloid concentration was about $5 \mathrm{E} 12$ particles per liter. About 20 times less than the lower zone of total colloids was found in water samples collected from the upper aquifer (around 2,511 feet of slanted depth), although colloid size distributions from these two zones appear to be rather similar.

Chemical analyses conducted by energy-dispersive $x$-ray spectroscopy (EDS) show that chemical elements such as $\mathrm{Al}, \mathrm{Si}, \mathrm{K}, \mathrm{Ca}$, and $\mathrm{Fe}$ are dominant in the colloid materials. From electron micrographs, varieties of colloid sizes and shapes have been illustrated. Lattice fringes obtained from the high-resolution transmission electron microscope (TEM) suggest that most colloids are crystalline materials. Diffraction patterns indicate that the colloids in the ER-20-5, \#3 Well are dominated by smectite and silica minerals. 


\section{INTRODUCTION}

Colloid was first referred to by T. Graham in 1861 as any suspension of material in a liquid where the suspension does not appear to separate even after a long period of time. The mechanism keeping the colloidal particles in suspension is the constant, yet random, thermal (Brownian) bombardment by the surrounding water molecules. Opinions vary somewhat, but the size range of colloidal particles is generally defined as several nanometers $(\mathrm{nm})$ to one micron. Particle sizes larger than one micron are usually difficult to keep suspended because Brownian motion is less able to keep them from settling downwards. Particles smaller than 3- to 5-nm are similar to the size of molecules and are considered as being essentially dissolved.

Conventionally, water samples were filtered through a 0.45 - (or $0.2-)$ micron $(\mu \mathrm{m}$-) size filter to remove solid materials. The distinction between solid and dissolved compounds in water is usually made by this membrane filtration. For example, whatever passes through the filter is considered to be dissolved materials. The filtration method, however, did not consider those particles smaller than 0.45 (or 0.2$) \mu \mathrm{m}$ in the filtered water. Recent measurements published in the literature suggest that a significant portion of the operationally defined dissolved materials is in fact in the form of colloidal particles. Colloids do not dissolve, but instead remain as a solid phase in suspension. Thus, colloid materials in a filtered water sample are operationally defined as particles between $3 \mathrm{~nm}$ and $450 \mathrm{~nm}$ in size.

Colloids may be important in regulating the concentrations and transportation of contaminants in groundwater. The reason to elevate contaminant concentration in water is because its high specific surface areas make these colloids efficient sorbents for contaminants. Contaminant transportation is enhanced because the colloidal material may suspend in solution and transport along with water movement. Radionuclides such as plutonium, americium, cobolt-60 and cesium- 137 have been demonstrated to be able to migrate farther and faster in groundwater environments than those that have been predicted by conventional models using sorption coefficients. Laboratory studies conducted by the Hydrologic Resources Management Program (HRMP) personnel using the filtration method show that these radionuclides are associated with colloidal materials in groundwater at the Benham underground nuclear explosion site.

In order to understand the potential for colloid-facilitated transport, we have conducted several tasks to characterize colloidal materials near Benham and other Nevada Test Site (NTS) groundwater samples. The objectives of these studies are (1) to quantify the total colloid concentration, (2) to evaluate colloid size distributions, and (3) to determine colloid shape and chemical and mineral composition.

Our groundwater colloid study is an ongoing effort. In this report, four major tasks were summarized. These were

\section{Colloid size and concentration analyses for the ER-20-5 groundwater sample, which had been stored for several months.}

II. The effects of the sample container, the storage method, and aging time on colloid stability using J13 water.

\section{Colloid characterization of groundwater samples collected freshly from ER-20-5, \#1 and \#3 wells.}

\section{Colloid analyses of Cheshire, U20n, groundwater samples.}

In Task I, we have analyzed colloids in samples that were used for filtration study during the summer of 1997 . Some samples had been filtered through a series of Millipore filters with pore sizes $50 \mathrm{~nm}$ and 100,000 nominal molecular weight (nmwl). Several sets of ER-2051-97-120 samples were obtained from HRMP personnel. These groundwater samples were collected from ER-20-5-1 Well near the Tybo and Benham underground nuclear explosion sites. In all, eight sets of samples from different filter-size fractions were studied. Five sets of the samples, (A) blank nanopure water, (B) 50-nm filter water, (C) $<50$-nm filter water, (D) 100,000-nmwl filter water, and (E) $<100,000$-nm wl filter 
water, were analyzed for colloid concentrations in this task. Three sample treatments, (1) undiluted, (2) 1:10 dilution, and (3) 1:100 dilution, were used for each set of samples. (Each set of samples had been prepared at full strength, at 1:10 dilution, and at 1:100 dilution.)

In Task II, we evaluated the effects of the sample container, storage, and aging time on colloid stability. In this study, J13 (NTS-TA25) well water was collected in both Teflon bottles and a 53-gallon (about 200-liter) barrel/drum made of polyethylene. Groundwater samples that were collected in Teflon bottles were transferred back to Los Alamos National Laboratory (LANL) for analyses within 24 hours. Water samples in the 53-gallon polyethylene barrel were transported to LANL by surface transportation. It took about two weeks to get to LANL from NTS, and the groundwater samples were analyzed for colloid 18 days after they were collected. The goals for this task were to check the colloid stability after it was sampled and to compare different sample containers. A 53-gallon (200-liter) barrel was selected because similar water storage and transportation processes were used in Task $\mathrm{L}$.

During Tasks I and II, we had developed and continually modified a colloid sampling procedure. This procedure is listed in sampling protocol section of this report. Using this protocol, HRMP personnel collected ER-20-5 Wells \#1 and \#3 samples on July 9, 1998, and on April 30, 1998, respectively. Samples from the ER-20-5 Well \#3 were analyzed for colloid within 24 hours after sampling. However, ER-20-5 Well \#1 was analyzed on July 13, 1998, which was four days after sampling due to transportation delay. In Task III, the colloid size distribution and total concentration in these freshly collected groundwater samples were studied. Colloidal materials from these wells were further analyzed by scanning electron microscope and transmission electron microscope (TEM) to study their chemical compositions, particle shapes, and mineral components.

In Task IV, we analyzed the colloid concentration and size distribution in Cheshire, U20n, water samples. Two sets of Cheshire samples were obtained from HRMP personnel. The first set was received and analyzed on July 30, 1998. This sample was collected at a depth of about 2,511 feet on July 28, 1998. A second set of samples from the Cheshire, U20n, well was collected at a lower interval (about 4,000 feet) which was labeled as PP\#1 DD-H on September 21, 1998. This sample was received and analyzed on September 28, 1998. To avoid confusing the sample collecting date with the analyses date, only collecting dates were reported in the results section.

\section{INSTRUMENTS}

\section{High-Sensitivity Liquid In Situ Particle Spectrometer}

Colloidal particles with sizes ranging from 50- to 200-nm are determined by a 16-channel high-sensitivity liquid in situ particle spectrometer, HSLIS-S50, manufactured by Particle Measuring System, Inc. (Boulder, Colorado). Details of instrument operation and design can be found from the instrument's operating manual (P/N 10167-4) and user's guide (P/N 10146-4) that are published by Particle Measuring System, Inc. A brief introduction of this instrument and a short description of its theory of operation are listed below. The instrument operates on the principle that light scattered by a liquid-borne particle resident in a laser beam is directly proportional to the particle size. It uses laser illumination with a high-resolution optical system, collecting particle-scattered light over a range of scattering angles at $\pm 40^{\circ}$. Sizing is accomplished "in situ" through pulse-height analysis of the light scattering. The instrument has a 50- to 200-nm size range. The laser used is a $30-\mathrm{mW}$ solid-state device (780-nm wavelength). The optical system includes cylindrical reflective condensing elements and spherical reflective collecting optics. The 0.85 numerical aperture of collection objective optics provides a large solid angle for collecting particle scattering light. All glass optical elements are antireflective coated. The laser beam is focused to the sample region using astigmatic condensing optics. Particles in the sample area reflect and obscure the laser light. The size and concentration of the particle is determined from the amount of reflected light. Optics focus light onto a photodiode detector that transmits electrical pulses to a pulse height analyzer board for analysis. The board sizes the particles into 16 size classes. The particle size and size range for each channel measured are listed in Table 1. 
TABLE 1. Particle Size Channel and Measured Size Range

\begin{tabular}{|c|c|c|}
\hline CHANNEL & $\begin{array}{c}\text { REPORTED } \\
\text { PARTICLE SIZE } \\
(\mathbf{n m})\end{array}$ & $\begin{array}{c}\text { MEASURED } \\
\text { PARTICLE SIZE } \\
\text { RANGE }(\mathbf{n m})\end{array}$ \\
\hline 1 & 50 & $50-60$ \\
\hline 2 & 60 & $60-70$ \\
\hline 3 & 70 & $70-80$ \\
\hline 4 & 80 & $80-90$ \\
\hline 5 & 90 & $90-100$ \\
\hline 6 & 100 & $100-110$ \\
\hline 7 & 110 & $110-120$ \\
\hline 8 & 120 & $120-130$ \\
\hline 9 & 130 & $130-140$ \\
\hline 10 & 140 & $140-150$ \\
\hline 11 & 150 & $150-160$ \\
\hline 12 & 160 & $160-170$ \\
\hline 13 & 170 & $170-180$ \\
\hline 14 & 180 & $180-190$ \\
\hline 15 & 190 & $190-200$ \\
\hline 16 & 200 & $>200$ \\
\hline
\end{tabular}

Instrument measurements of colloid size and concentration were downloaded to a computer using the spreadsheet program Excel. Sample dilution and injection rates were incorporated with the calculation using the Excel files. Total colloid concentration between the 50- and 200-nm size range was determined by adding the measurements from all 16 channels. The final results of colloid particle counts versus colloid particle sizes were plotted using the computer Cricket Graph program.

\section{Electron Microscopes}

A scanning electron microscope (JEOL, 6300-FXV) with an x-ray energy dispersive spectrometer (EDS) system was used for imaging and elemental analysis. A Philips CM-30 analytical electron microscope (AEM) that is equipped for $\mathrm{x}$-ray microanalysis, electron diffraction, and conventional imaging was used for imaging colloid sizes, shapes, and diffraction patterns and for producing elemental composition analyses. A JEOL 6300FX field emission scanning electron microscope (SEM) with EDS was used for colloid imaging and elemental analyses. A JEOL 3000 field emission, high-resolution TEM (HRTEM) was used to image colloid shape and lattice fringes. These electron microscopes are located in the Electron Microscopy Laboratory, at the Material Science Laboratory, LANL.

For colloid samples used for electron were microscope study, we collected the colloids by filtering the groundwater sample through a nylon filter. The colloids were then resuspended and deposited onto an electron microscope sample grid. To avoid the precipitation of dissolved ions during the drying process, filtered samples were washed with deionized water. In ER-5-1 samples (Figures 1 to 5), it appears that all diluted samples contain a total colloid concentration of between $1 E 7$ and $2 E 7$ particles/mL. Furthermore, similar colloid size distributions were found in all diluted samples (Figures 6 to 10). 


\section{GROUNDWATER SAMPLING PROTOCOL}

We have developed a general sampling protocol to collect water samples for colloid study. The procedures are listed as follows:

1. Wash the Teflon bottles with detergent and rinse with low colloid nanopure water thoroughly to prepare the clean container for sampling.

2. Prepare the 0.2 - or $0.4-\mu \mathrm{m}$ nylon filter by rinsing and flushing the filter with low colloid nanopure water.

3. Check the colloid concentration using the HSLIS-S50 colloid instrument.

4. Transfer as soon as possible to the lab for sample dilution and filtration once the groundwater sample is collected from the well. Each sample container is labeled with its unique identifier (or bar code), sampling well name, brief description of sampling condition, date and time of collection, and collector's name.

5. Store the collected samples in a cooler, which is filled with ice (or blue ice). Keep the cooler in the shade.

6. Transport the water sample to the lab for colloid analysis within 24 hours (or as soon as possible).

\section{RESULTS AND DISCUSSION}

\section{Task I. Colloid Size Distribution and Concentration Analyses for ER-20-5-1 Samples}

We received eight sets of ER-20-5-1 (Barrel \#2) samples in July 1997. These samples were collected by Mr. Bennie Martinez from a filtration study that was conducted by Annie Kersting of Lawrence Livermore National Laboratory during the summer of 1997. Groundwater samples were labeled as ID:2051-97-120. We analyzed five sets of samples for colloid size distribution and total concentration. These five sets of samples are labeled as (1) L-5, blank nanopure water, (2) L-50F, 50-nm filter sample, (3) L-3, <50-nm sample water, (4) L-6F, 100,000-nmwl filter water, and (5) $\mathrm{L}-4,<100,000-\mathrm{nmwl}$ sample water. Within each set of samples, there are three subsamples, which are labeled as (A) undiluted, (B) 1:10 dilution, and (C) 1:100 dilution. In undiluted samples, the water was prepared in full strength. For 1:10 and 1:100 dilutions samples, the water samples were diluted 10 and 100 times, respectively, from their original concentration. Deionized water from TA48-28-101 was used for dilution.

Results of total colloid concentration in these filtered water samples are shown in Figures 1 to 5 . Colloid size distributions for these samples are shown in Figures 6 to 10.

Total colloid concentration results (Figures 1 to 6) show that all samples, including the nanopure water samples, contain large quantities of colloids. Because the HSLIS-S50 instrument can only measure colloid particles between 50and 200-nm in size, we should not find any colloids in those filtrate water samples that had filtered through a 50-nm (or smaller size) filter. However, we found millions of colloidal particles in sample sets such as L-3, L-4, and L-6F. Although undiluted blank nanopure water samples (code:L-5-A) contained the least amount of colloids; they still reached 3.3 million colloidal particles per milliliter $(\mathrm{mL})$. From the results of colloid analyses in those five sets of 
ER-20-5-1, Barrel \#2, ID:2051-97-120

Blank Nanopure Water (code: L-5)

$A=$ undiluted, $B=1: 10$ dilution, $C=1: 100$ dilution

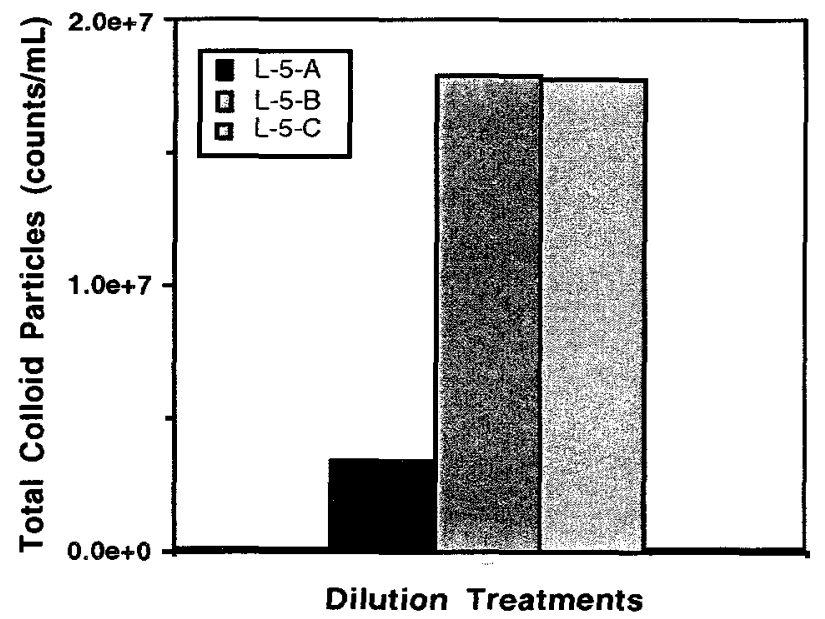

Figure 1. Total colloid concentration for blank nanopure water, L-5.

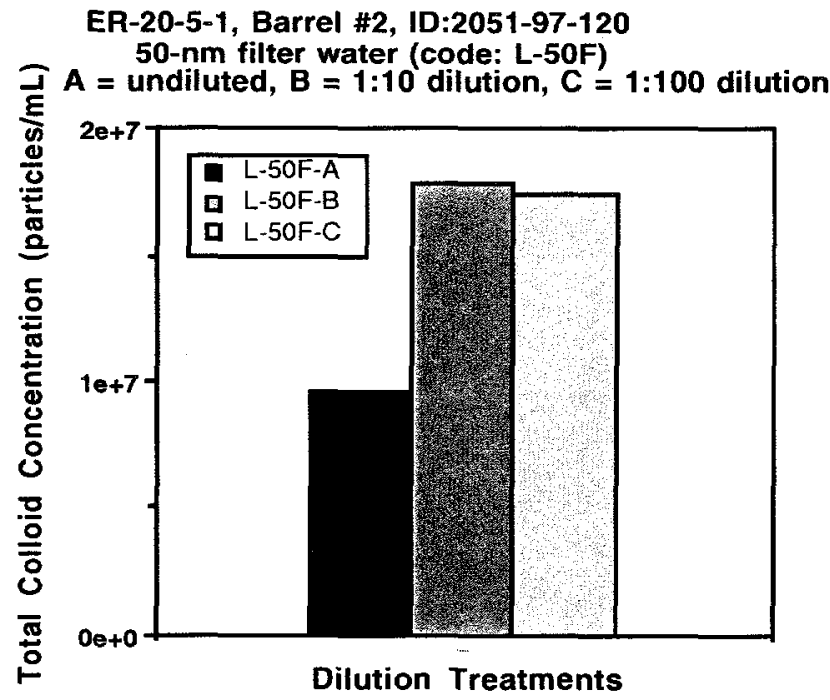

Figure 2. Colloid concentration for $\mathrm{L}-50 \mathrm{~F}, 50-\mathrm{nm}$ filter sample. 


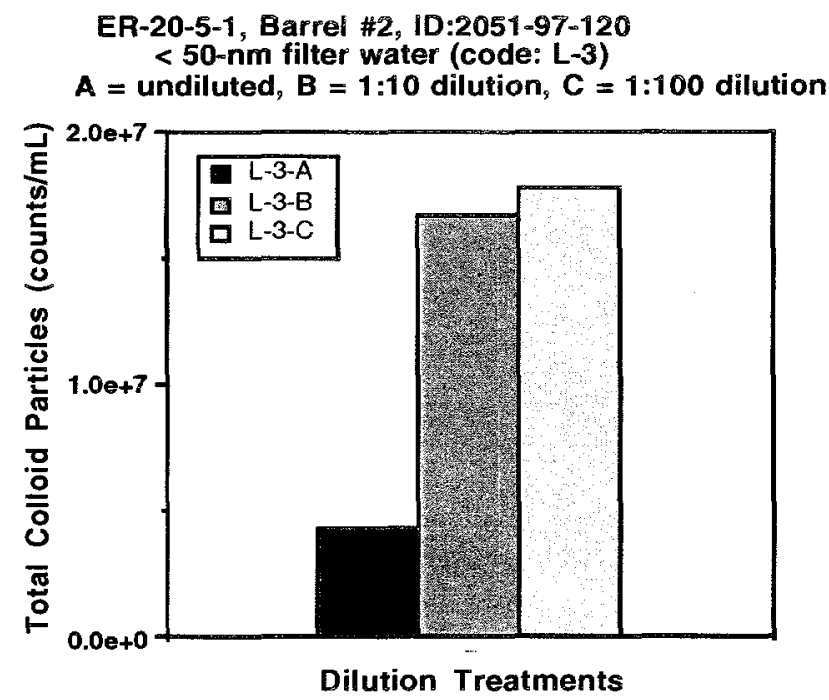

Figure 3. Colloid concentration for L-3, $<50-\mathrm{nm}$ sample water.

ER-20-5-1, Barrel \#2, ID:2051-97-120

$100,000-\mathrm{nmwl}$ filter water (code: L-6F)

$A=$ undiluted, $B=1: 10$ dilution, $C=1: 100$ dilution

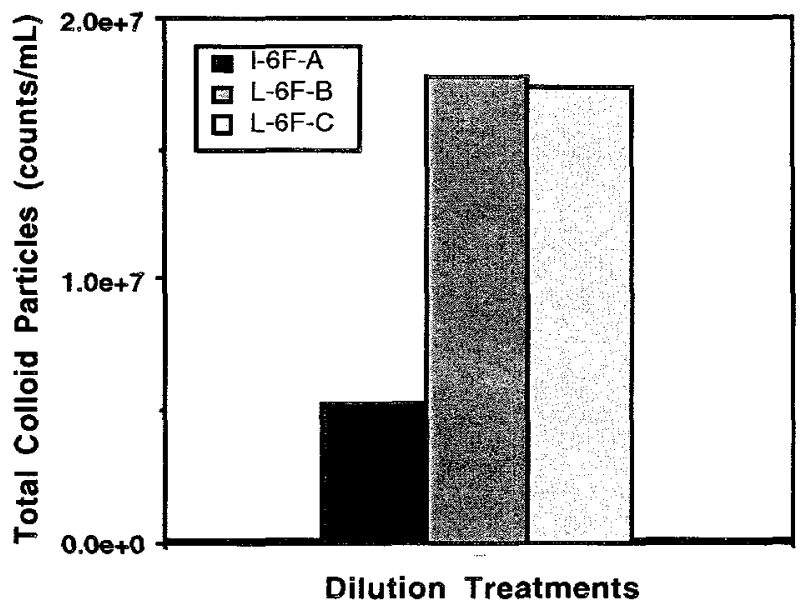

Figure 4. Colloid concentration for L-6F, 100,000-nmwl filter water. 
ER-20-5-1, Barrel \#2, ID:2051-97-120

$<100,000-\mathrm{nmwl}$ filter water (code: L-4)

$A=$ undiluted, $B=1: 10$ dilution, $C=1: 100$ dilution

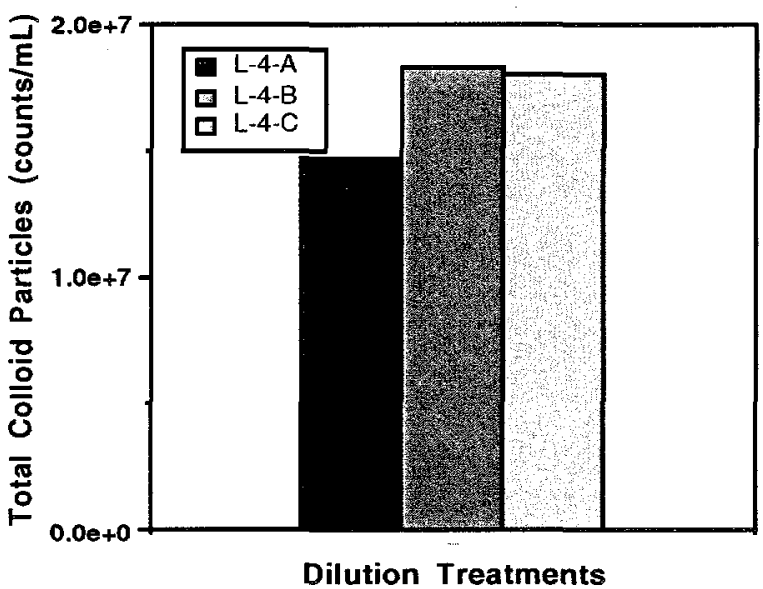

Figure 5. Colloid concentration L-4, $<100,000-n m w l$ sample water.

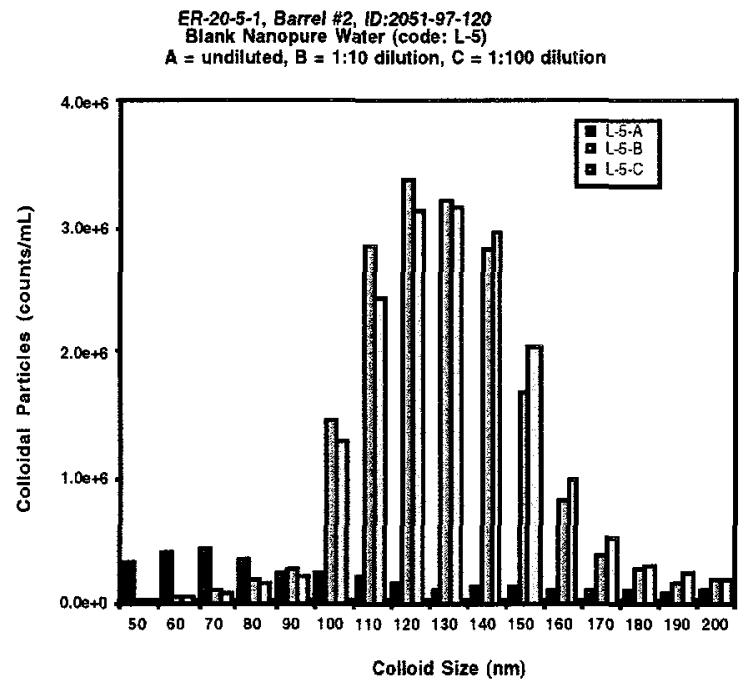

Figure 6. Colloid size distribution for blank nanopure water. 


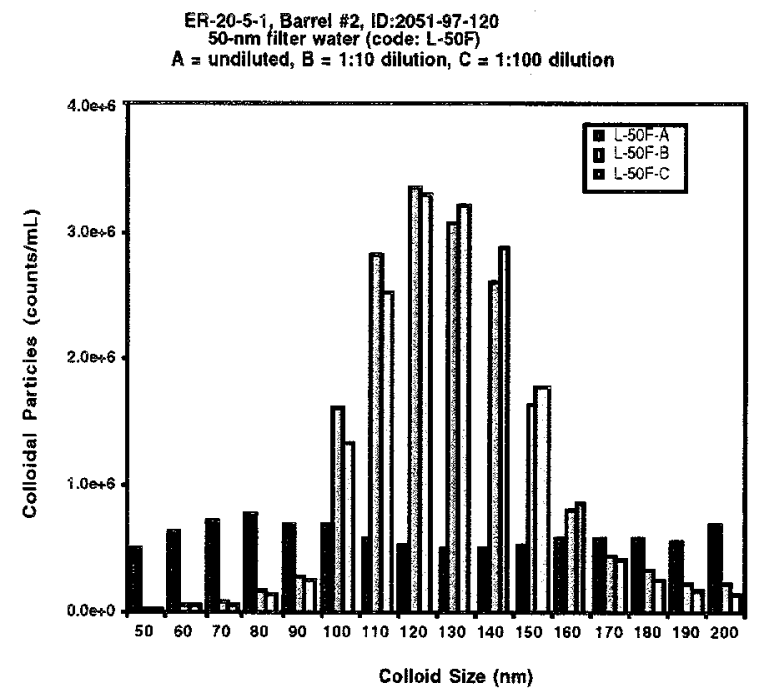

Figure 7. Colloid size distribution for 50-nm filter water.

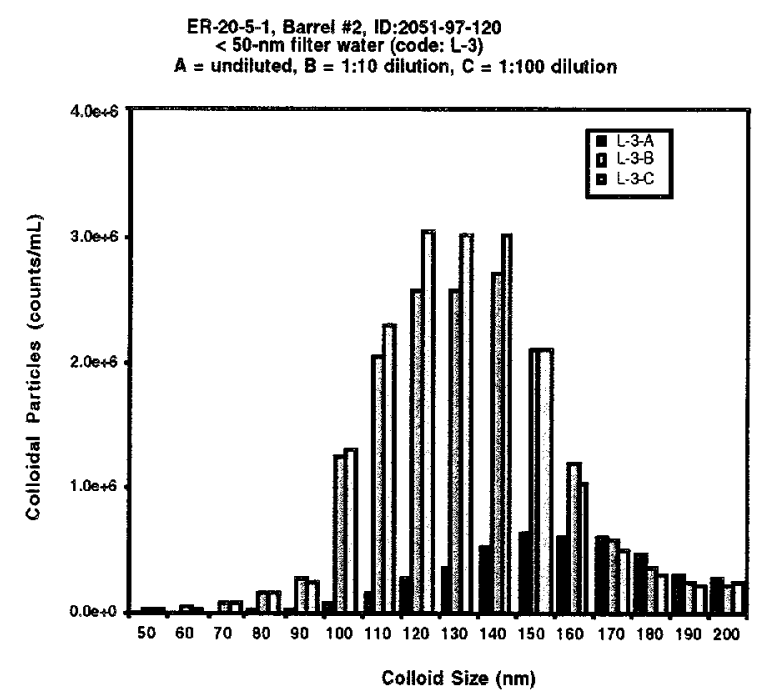

Figure 8. Colloid size distribution for $<50-\mathrm{nm}$ nmwl filter water. 


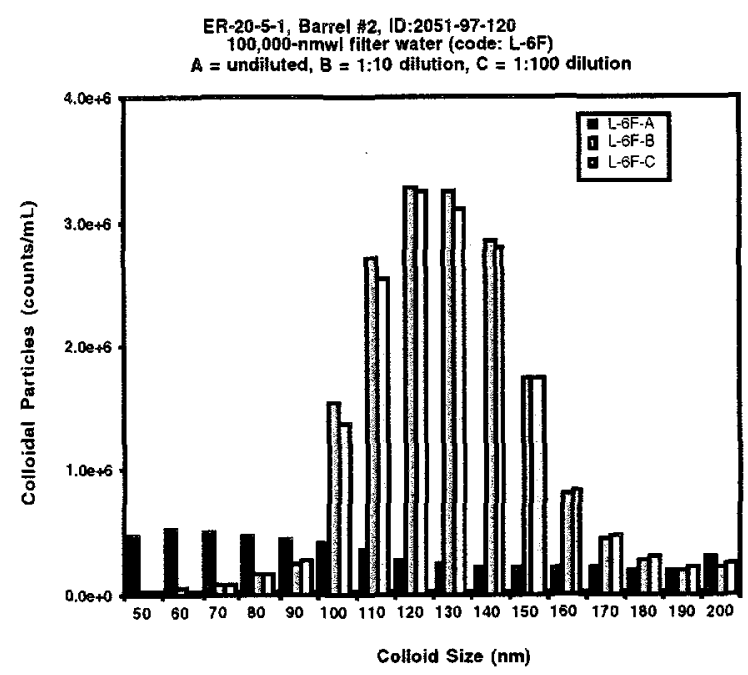

Figure 9. Colloid size distribution for 100,000-nmwl filter water.

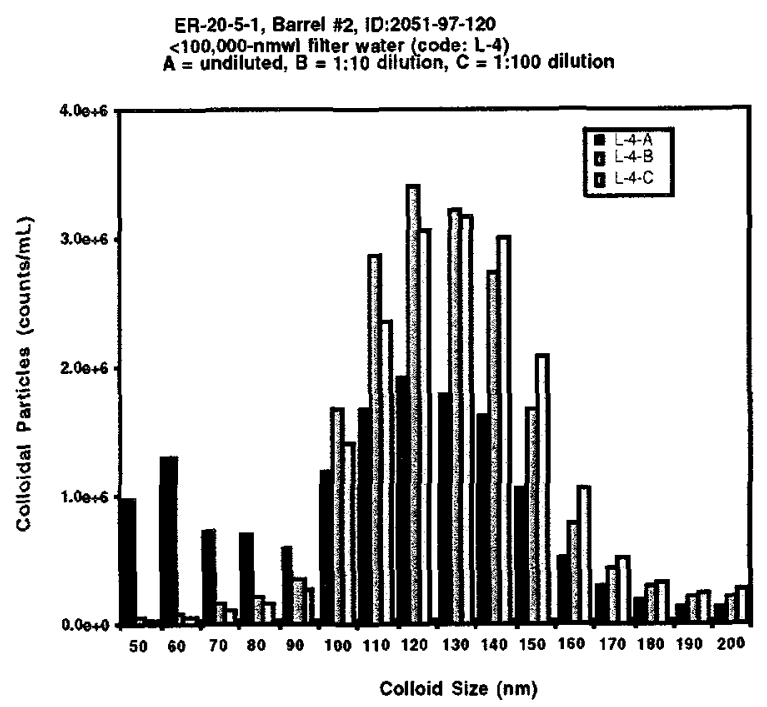

Figure 10. Colloid size distribution for $<100,000-\mathrm{nmwl}$ filter sample. 
It is also observed that samples after dilution treatments contain more colloids than undiluted samples. These results are unexpected because diluted samples should contain less colloids than undiluted samples. Obviously, alien colloidal materials have been introduced into the water samples during the dilution and/or filtration processes.

One source for the introduction of colloidal materials into the diluted samples is the labware that has been used for the dilution and/or filtration process. Labware such as pipettes, funnels, and containers, which are chemically clean, may not necessarily mean that they contain no colloidal materials. Another possible source of colloids is the water that was used in the dilution and cleaning processes.

Colloid particles may be present in high concentrations in nanopure water if it is not stored properly. For example, nanopure water samples were collected from the lab where the dilution was performed. One water sample was collected from a 25-gallon water storage bottle in the lab at TA-48-28-101. The water in this storage bottle was used for all the dilution processes in these ER-20-5 groundwater samples. A second sample was collected directly from the outlet of the nanopure water system at the same lab (i.e., without being poured into the storage bottle). A third sample was collected from the nanopure water in TA-48-1-304 that was identified as containing low colloidal material. In these experiments, all containers for collecting water samples were precleaned according to cleaning processes listed in the previous section and monitored for colloid contents by the HSLIS-S50 instrument. It should be noted that the water-generating system in room TA-48-1-304 contains both a $0.2-\mu \mathrm{m}$ filter, which is part of the nanopure deionization water system and an external $0.05-\mu \mathrm{m}$ filter. The colloid content in the water generated from TA-48-1-304 is constantly monitored by the HSLIS-S50 system, and this water was used for cleaning all the labwares.

Figures 11 and 12 show the results of colloid in these water samples. Almost six hundred thousand (6E5) colloids per $\mathrm{mL}$ appeared in the storage bottle water. Water samples collected directly from the nanopure system in TA-48-28-101 contain more than fifty thousand (5E4) colloids $/ \mathrm{mL}$. The least amount of colloids (fifty-six hundred colloids/mL) was found in the water system at TA-48-1-304. These results illustrate that colloids were present in the water that had been used for the dilution processes. It was apparent that the source of dilution water for the ER-20-5 samples contaminated them with colloids. Thus, only low-colloid water should be used for cleaning and dilution processes for colloid analyses.

An experiment was conducted to check the potential of colloid contamination generated by filter paper and labware used for filtration. In this study, a $47-\mu \mathrm{m}$ diameter, 0.45 micron pore-size filter (made by Nuclepore) with a plastic holder was evaluated for colloid generation. The filter holder itself was rinsed with low-colloid-content nanopure water three times before the filter was installed. About $150 \mathrm{~mL}$ of nanopure water was used to be filtered through the filter. The filtrate was collected in a clean container and analyzed for total colloid concentrations. Results show that about 4E6 colloidal particles $/ \mathrm{mL}$ were generated from the system (a $47-\mu \mathrm{m}$ diameter, 0.45 micron pore-size filter with a precleaned holder). Apparently, colloids do generate from the filter material.

We have also evaluated filters that were made of cellulose acetate, nylon, cellulose nitrate, cellulose esters, polyethersulfone, and polycarbonate. Results show that all filter materials will generate a large quantity of colloids if they have not been rinsed carefully. For example, more than five million colloids appeared in the filtrate after filtering low-colloid-content nanopure water through a cellulose acetate $0.2-\mu \mathrm{m}$ filter. One possible reason for the presence of colloidal materials in the filtrate is that most of the filters were designed and made for biological application. Some filters are even treated with surface activating agents and/or other chemicals to enhance their performance. For example, surfactants and polymers have been added or coated in some filters as wetting agents. The performance of some filters, which are used for sterilization purposes, is determined only by evaluating the number of biological particles of interest (i.e., bacteria) filtered through the system. Therefore, it is not surprising to have colloidal material (organic surfactants and polymers) in the filtrate if the filter was not thoroughly washed. Nevertheless, from these filter studies, we found that nylon filters produce the least quantity of colloids after washing and flushing three times with nanopure water. Therefore, we have selected nylon filters for all sample filtration purposes. 


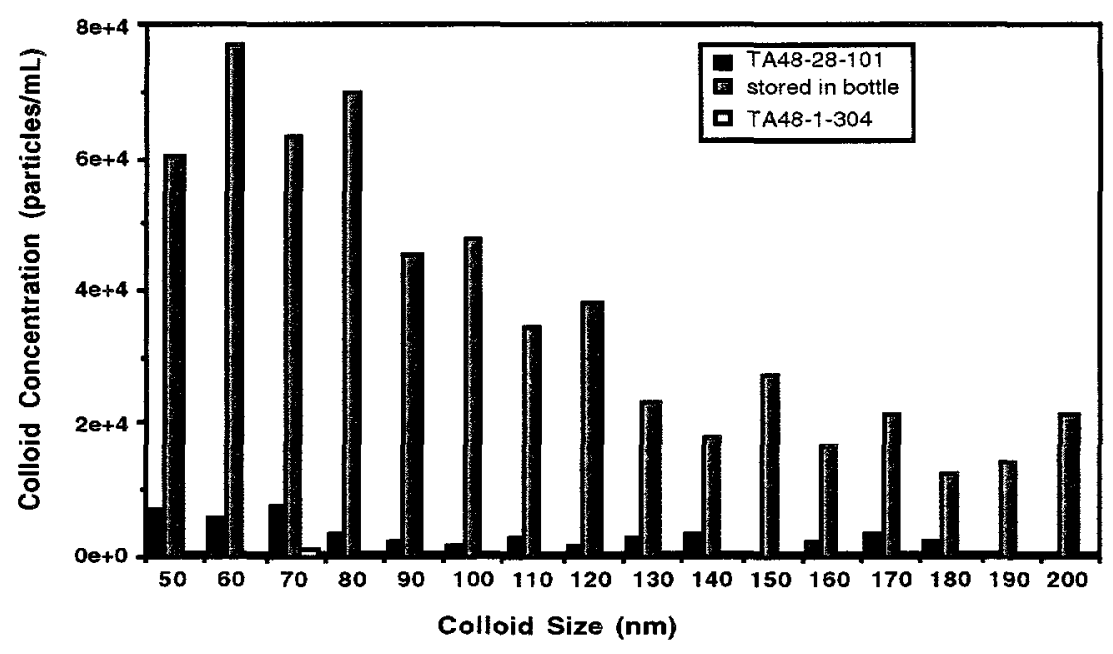

Figure 11. Colloid size distribution for nanopure water samples.

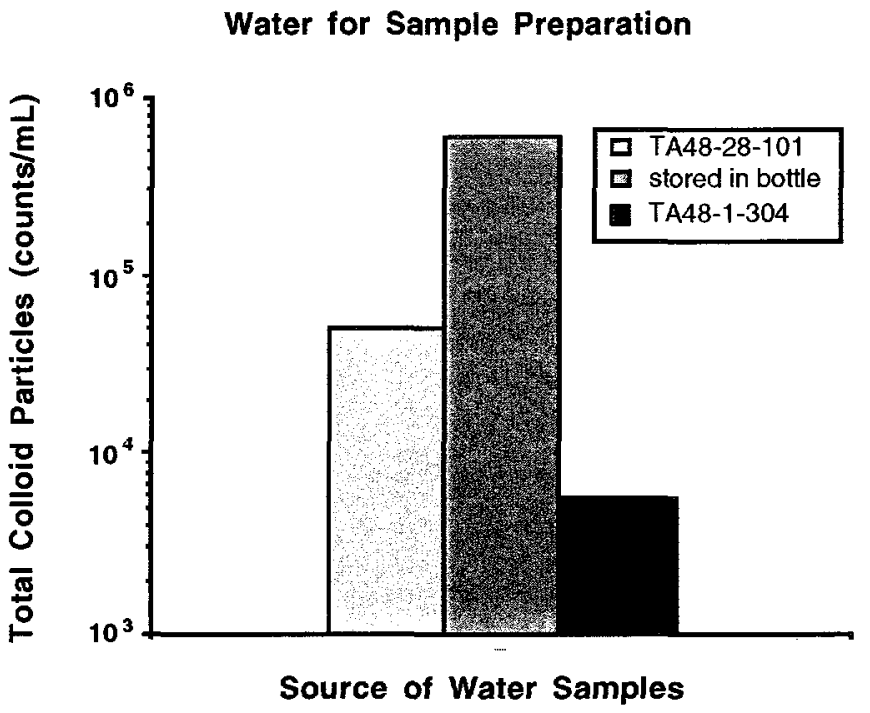

Figure 12. Total colloid concentrations for nanopure water samples. 
Task II. Effects of the Sample Container, Storage, and Aging Time on Colloid Stability

Besides the filter and dilution water, conceivable factors that might affect the colloid concentration and size distribution for samples analyzed in Task I are sample storage and aging time. Groundwater samples in Task I were originally collected in a 53-gallon (200-liter) barrel. In addition, we analyzed the sample in Task I for colloid concentration and size distribution several months after they were collected. Therefore, in Task II, the effects of sample container, storage, and aging time on colloid stability were evaluated.

In this study, we have selected $\mathrm{J} 13$ groundwater as a surrogate because $\mathrm{J} 13$ water is readily available and has been studied extensively by Yucca Mountain Project (YMP) personnel. In addition, J-13 water is similar to other NTS groundwaters in having relatively low ionic strength, a property important in colloid stability. It should be noted that we learned later in Task III that J13 water has a much lower colloid concentration than the ER-20-5 Wells. We have also evaluated the stability of colloids in groundwater once the water sample was brought to LANL. Several types of containers and treatments were used for comparison. Two sets of J-13 water samples were collected in a precleaned Teflon bottle and a 53-gallon (200-liter) polyethylene barrel, respectively. The third set of water samples was collected in Teflon bottles after the water sample was filtered through a precleaned nylon filter with $0.2-\mu \mathrm{m}$ pore size.

The samples, which were stored in Teflon bottles, were transferred back to the lab for colloid analyses within 24 hours after they were collected in the field. It was done by hand, carrying all samples and flying back to Los Alamos on the same day as sampling took place. The second set of J13 water samples, which was stored in a 53-gallon (200-liter) polyethylene barrel, was transferred back to Los Alamos by surface transportation. This sample was analyzed 18 days after it was collected. Soon after the 200-liter plastic barrel arrived in Los Alamos, part of the water sample was transferred from the barrel to Teflon bottles for colloid analyses. The water sample that was stored and transported in a 200 -liter barrel was also studied by filtering through a precleaned nylon filter with $0.2-\mu \mathrm{m}$ and $0.45-\mu \mathrm{m}$ pore sizes in the lab.

Figures 13 to 15 show the results of colloid size distribution and total colloid concentration for samples stored in the Teflon bottles and in the 200-liter plastic barrel. Unfiltered water samples that were stored in the 200-liter barrel or Teflon bottles are compared in Figure 13. Results suggest that more colloids are present in the sample that was stored in the 200-liter barrel than in the sample stored in the Teflon bottle. The colloid particle size distributions for these two sets of samples were also different. For the water sample that was stored in the 200-liter barrel, results indicate that the smaller the colloid in size, the higher the colloid in concentration. This result infers that some colloids might have been introduced into the water sample by the 200 -liter plastic barrel. It should be noted that unlike the Teflon bottles that had been cleaned in the lab, the 200-liter barrel was not precleaned in the lab, although it has been rinsed with J-13 water before the water was collected.

After the barrel-stored sample was filtered through a $0.2-$ or $0.45-\mu \mathrm{m}$ nylon filter, the colloid concentration decreased significantly (as shown in Figure 14). The data show that about three-quarters of the colloids were lost during the filtration process by using a $0.2-\mu \mathrm{m}$ nylon filter. From the particle size distribution, it was found that the most noticeable changes were the reduction of colloids among relative larger sizes (130- to 200-nm) of colloidal particles. Figure 15 shows that there are few colloids left between sizes 200- and 130-nm after filtration. These results indicate that colloidal particles that are smaller than the filter size would be filtered out of the filtrate. Although no visible material was found on top of the filter during the filtration process, the filter was still able to trap colloids smaller than the filter pore size. 


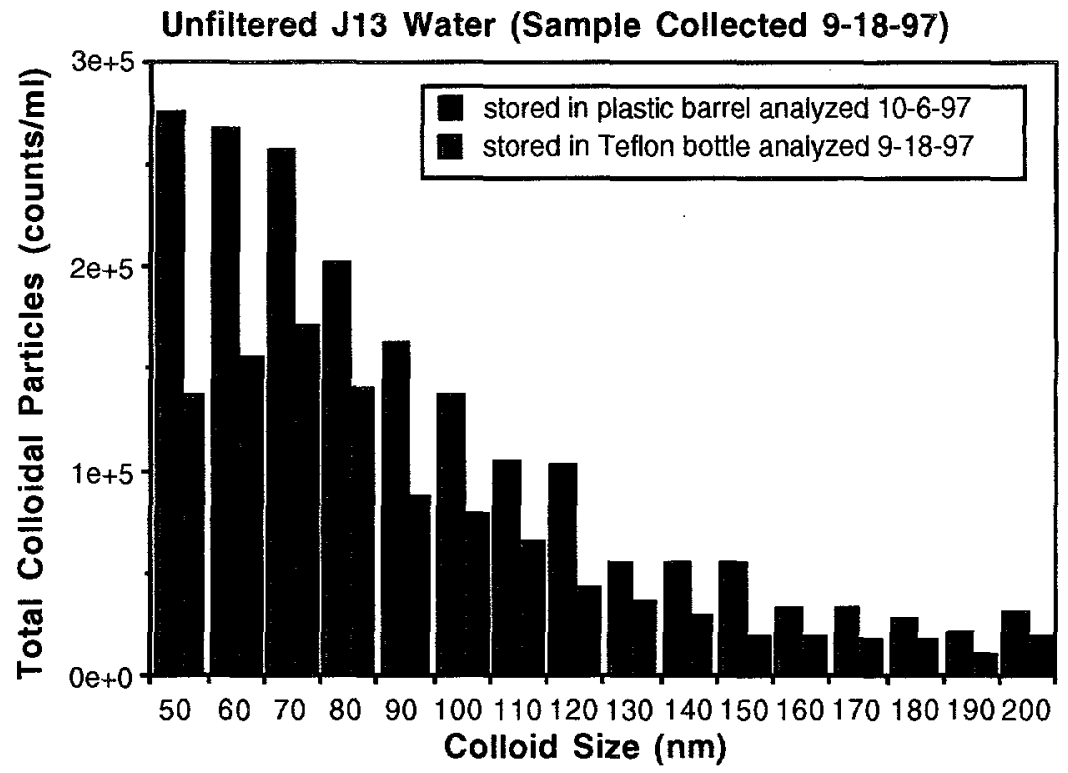

Figure 13. Colloid size distribution for J13 water stored in different types of containers.

$\mathrm{J} 13$ ground water Stored in 200-Liter Barrel (collected 9-18-97, analyzed 10-6-97)

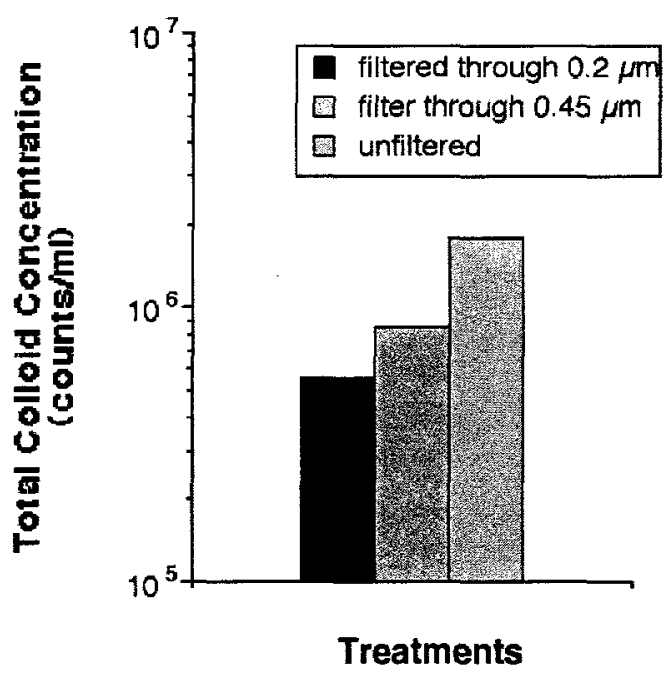

Figure 14. Total colloid concentration between the sizes of 50 and $200 \mathrm{~nm}$ in J13 water samples with and without filtrations. 


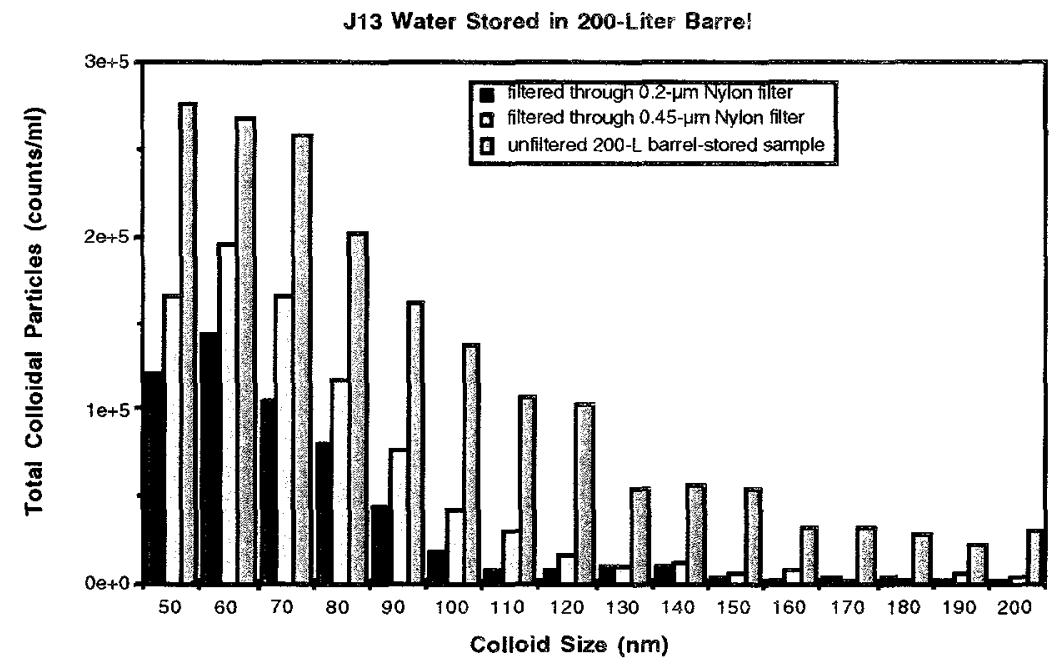

Figure 15. Colloid size distribution for $\mathrm{J} 13$ water stored in a 200 -liter barrel and filtered through $0.2-$ and $0.45-\mu \mathrm{m}$ nylon filters.

Although results suggest that the 200 -liter barrel may introduce alien colloids into the water sample, it is not clear whether the delay of analyses for about two weeks because of transportation from the field to the lab can cause any colloid concentration and size distribution changes. Furthermore, it is not clear whether filtration processes will generate any changes in the colloid size distribution and concentrations or not. For these reasons, the filtered and unfiltered J13 water samples, which were taken from the 200-liter (53-gallon) barrel, were further studied for their short-term colloid stability.

In this study, samples were monitored for water chemistry such as $\mathrm{pH}$, Eh, and oxygen content, colloid concentration, and size distribution once they were exposed to the lab environment for about 10 days. To conduct this experiment, samples were loosely covered (allowing the degas process to proceed faster). To avoid the evaporation of the water samples, all sample containers were stored inside a large beaker, which was filled with deionized water. In this experimental condition, a relatively high humidity was maintained inside the beaker. Results of colloid concentration and size distribution for filtration treatments are shown in Figures 16 to 19.

For comparison, water chemistry data are reported in Table 2. The slight decrease in dissolved oxygen after filtration was probably due to the low vacuum applied to facilitate the filtration process. However, all dissolved oxygen levels reached $6.5 \mathrm{mg} / \mathrm{L}$, or above, after 9 days. Redox potential was measured by using a platinum electrode with $\mathrm{Ag} / \mathrm{AgCl}$ reference. The Eh value went up from $210 \mathrm{mV}$ to $294 \mathrm{mV}$ in 9 days. This is probably due to the increase of oxygen content. For water $\mathrm{pH}$ values, they all reached 7.9 or above in three days. This is probably due to the decrease insoluble carbon dioxide content. 


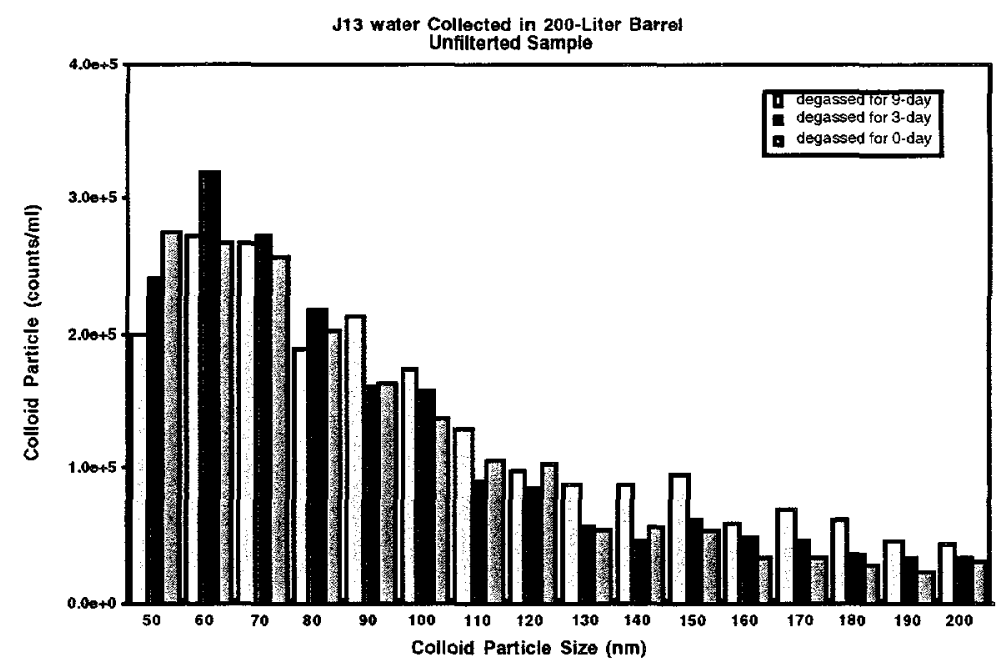

Figure 16. Colloid size distribution for unfiltered J13 water stored in Teflon bottles before and after being degassed.

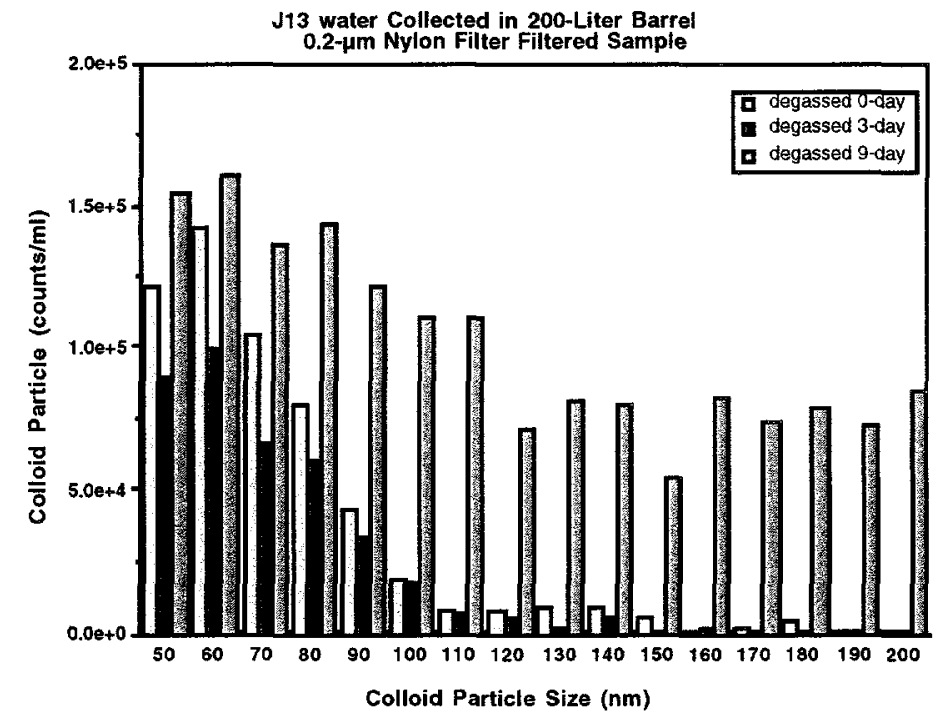

Figure 17. Colloid size distribution for $0.2-\mu \mathrm{m}$ filtered $\mathrm{J} 13$ water stored in a Teflon bottle before and after being degassed. 


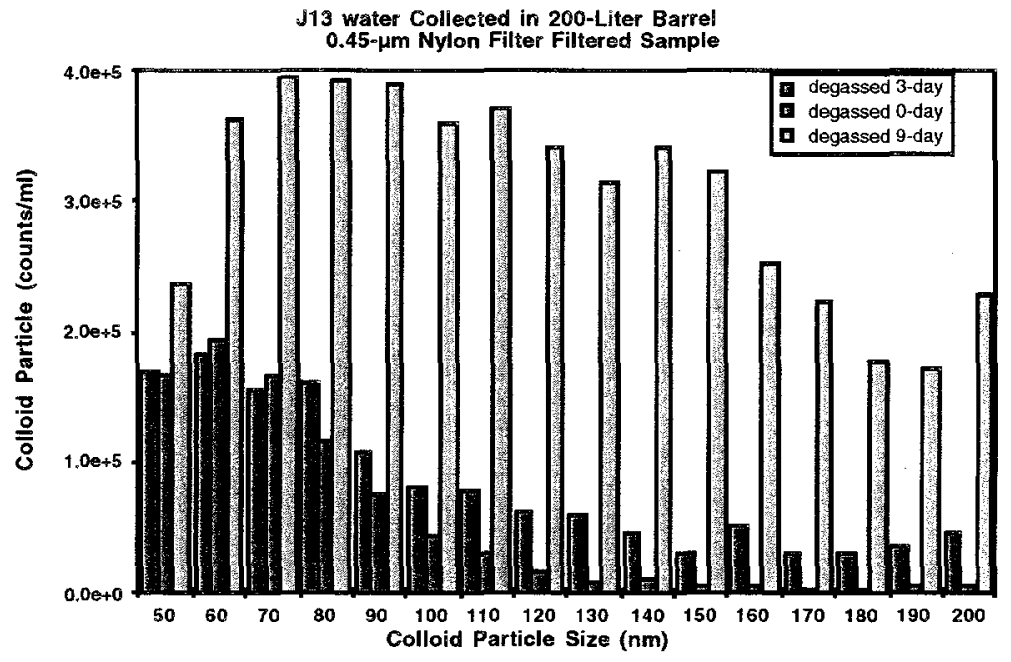

Figure 18. Colloid size distribution for $0.45-\mu \mathrm{m}$ filtered $\mathrm{J} 13$ water stored in a Teflon bottle before and after being degassed.

Figure 16 shows the colloid size distribution of unfiltered samples. Colloid concentration remains pretty much the same after 9 days. However, for 0.2 - and $0.45-\mu \mathrm{m}$ filtered samples, colloid concentration increased dramatically, especially for sizes between 100 to $200 \mathrm{~nm}$ (as shown in Figures 17 and 18). Apparently, new colloid will be produced and/or small-sized colloids become aggregated. These results suggest that colloids are not stable in water samples after 9 days of degassing.

Table 2. Water Chemical Properties of J13 Groundwater.

\begin{tabular}{|c|c|c|c|c|c|}
\hline & Day & $\mathrm{Eh}(\mathrm{mV})$ & $\mathrm{pH}$ & $\begin{array}{c}\text { Dissolved } \\
\text { Oxygen } \\
(\mathrm{mg} / \mathrm{L})\end{array}$ & Temp ${ }^{\circ} \mathrm{C}$ \\
\hline \multirow{3}{*}{ Unfiltered Water } & day 0 & 210 & 7.2 & 6 & 19.7 \\
\hline & day 3 & 295 & 7.9 & 6.3 & 21 \\
\hline & day 9 & 293 & 8.1 & 6.7 & 21.6 \\
\hline \multirow{3}{*}{$0.45 \mu \mathrm{m}$ Fïltered } & day 0 & 211 & 7.4 & 5.2 & 20.1 \\
\hline & day 3 & 295 & 8.1 & 6 & 21 \\
\hline & day 9 & 294 & 8.3 & 6.7 & 21.6 \\
\hline \multirow{3}{*}{$0.2 \mu \mathrm{m}$ Filtered } & day 02 & 12 & 7.7 & 5.2 & 20.1 \\
\hline & day 3 & 295 & 8.1 & 6 & 21 \\
\hline & day 9 & 292 & 8.3 & 6.5 & 21.6 \\
\hline
\end{tabular}




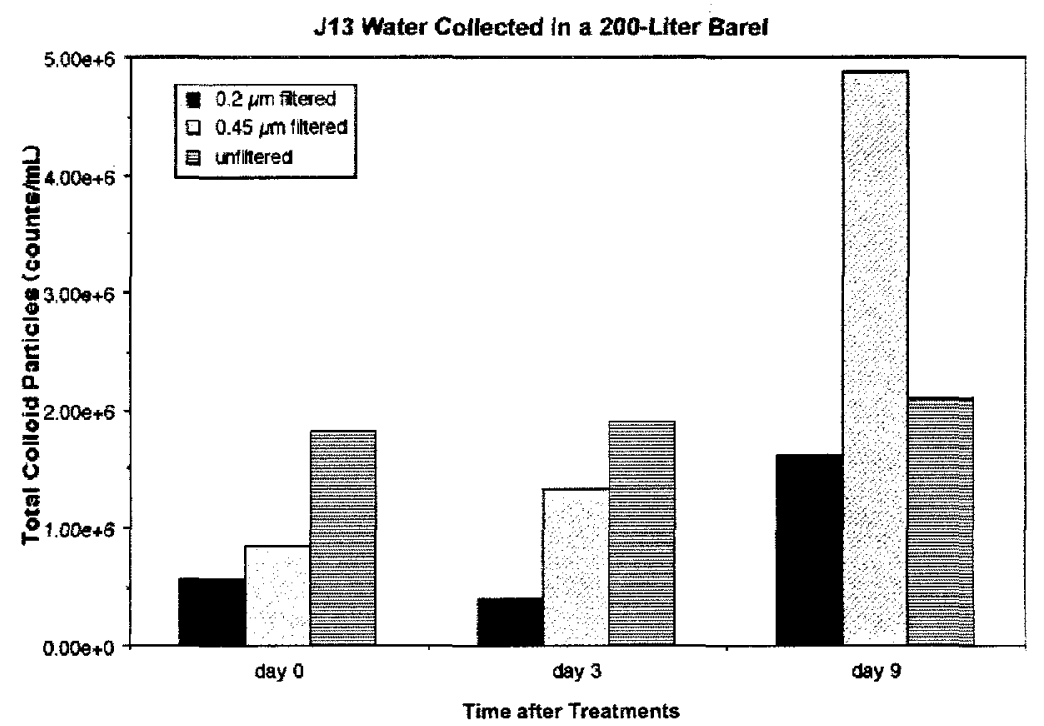

Figure 19. Colloid concentrations in filtered and unfiltered J13 barrel samples after degassing in a Teflon bottle.

To evaluate the effect of long-term sample storage on colloid stability, we stored the J13 water samples in the 200-liter barrel for about six months. Filtered and unfiltered J-13 water samples were also stored in Teflon bottles. One bottle was submerged in a water bath to prevent the degassing process during the 6-month storage period. The objective was to find out the colloid stability for an aged water sample in a closed environment. The results of the 190-day sample storage in different conditions on colloid size distributions are presented in Figures 20 to 24.

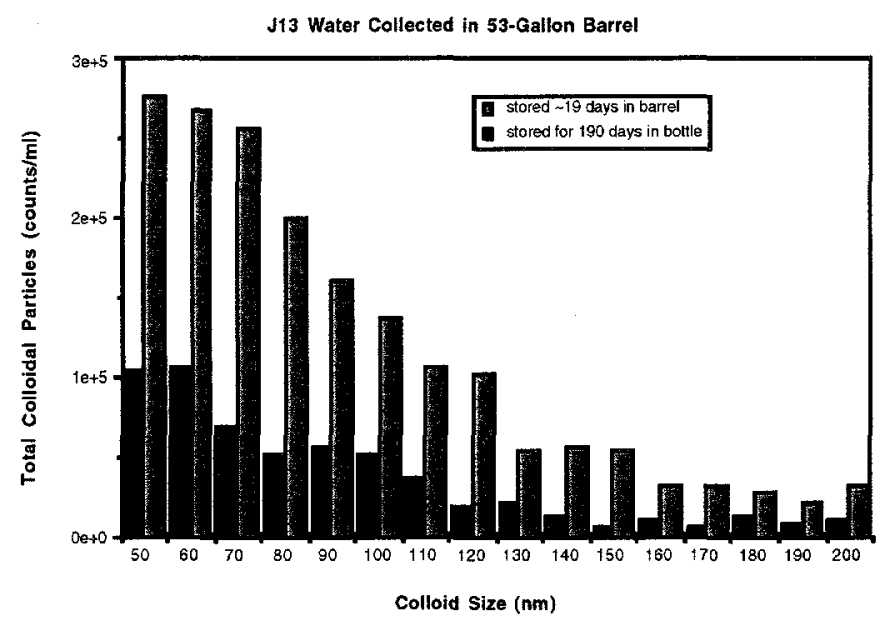

Figure 20. Colloid size distribution for unfiltered J13 water before and after being stored in a Teflon bottle for 190 days. 
Colloid size distribution of unfiltered water after 190 days storage is shown in Figure 20. It appears that the colloid concentrations were reduced in all particle sizes. No significant difference was found for water samples stored in Teflon bottles with or without being submerged in a water bath (Figure 21). The colloid size distribution changed for samples that had been filtered through 0.2 and $0.45-\mu$ m nylon filter (Figure 22 and 23 ). In this treatment, the concentration of large-sized colloidal particles ( 200 to $100 \mathrm{~nm}$ ) increased; meanwhile, the simall-sized particles (50 to $90 \mathrm{~nm}$ ) decreased.

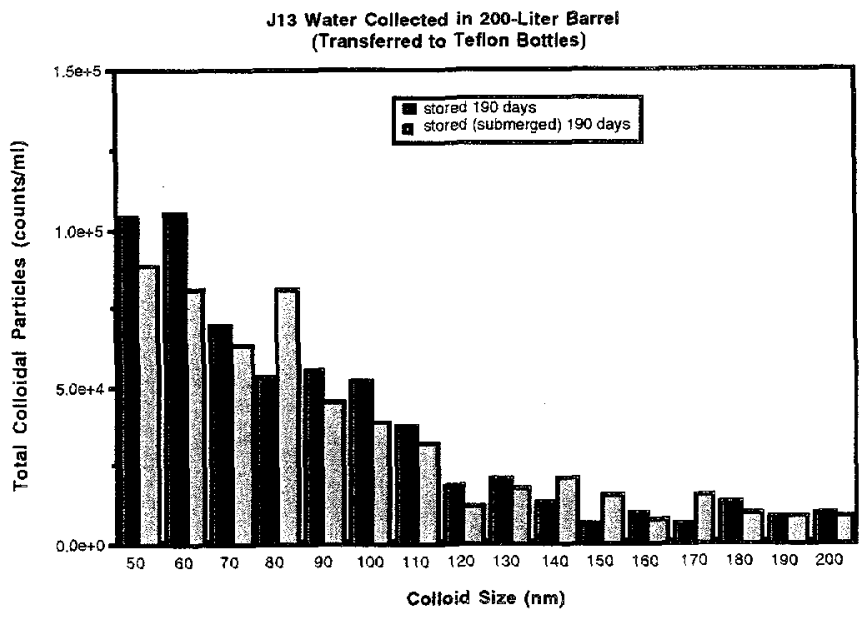

Figure 21. Colloid size distribution for unfiltered $\mathrm{J} 13$ water before and after being stored in a Teflon bottle for 190 days.

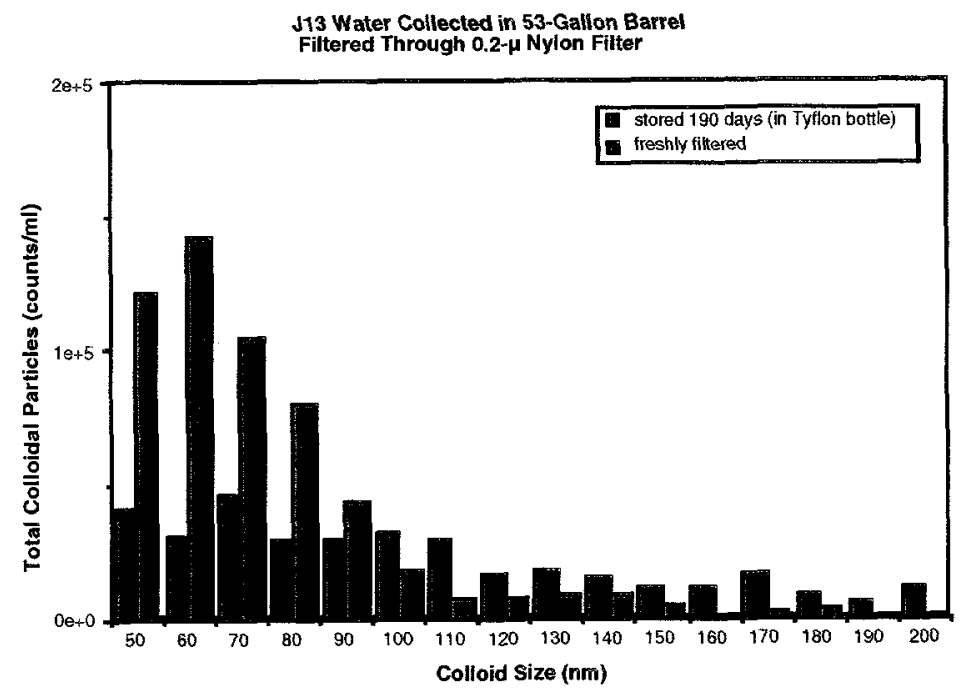

Figure 22. Colloid size distribution for $0.2-\mu \mathrm{m}$ filtered $\mathrm{J} 13$ water before and after being stored in a Teflon bottle for 190 days. 


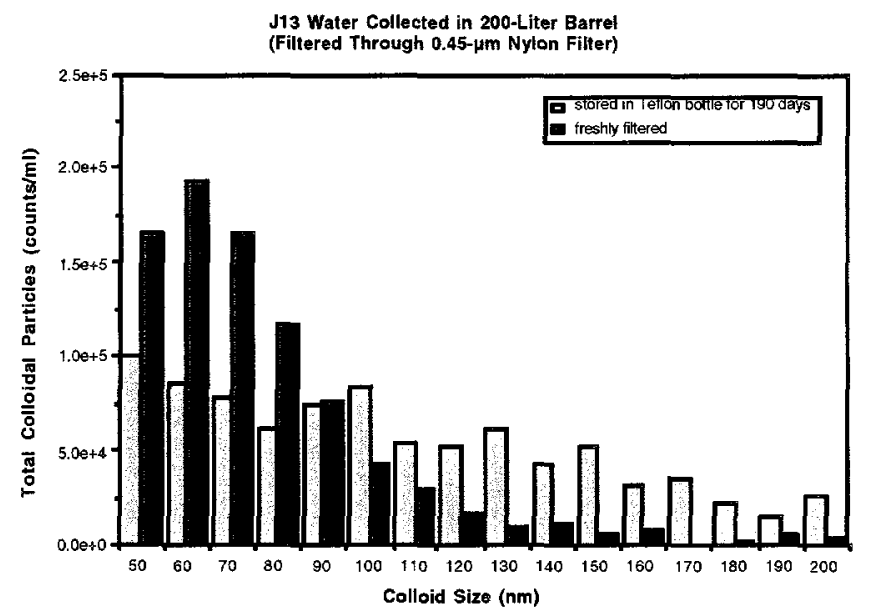

Figure 23. Colloid size distribution for $0.45-\mu \mathrm{m}$ filtered $\mathrm{J} 13$ water before and after being stored in a Teflon bottle for 190 days.

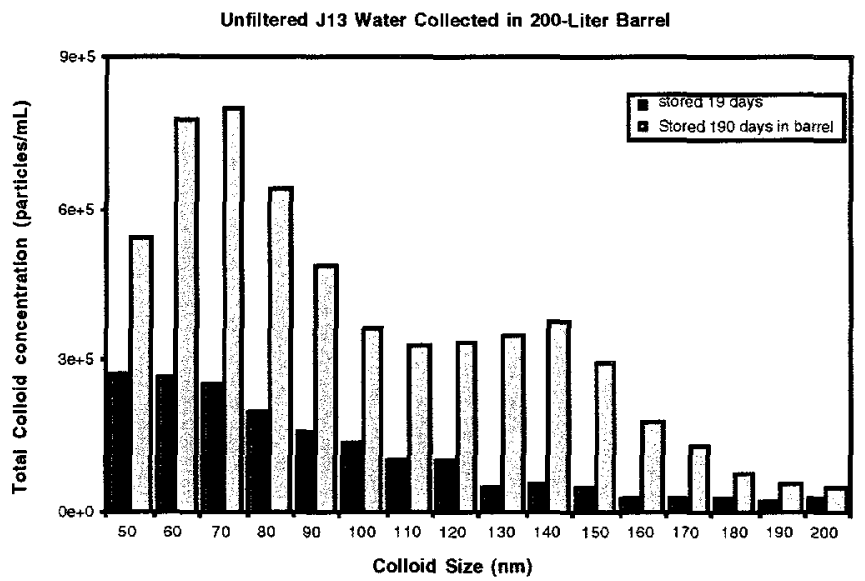

Figure 24. Colloid size distribution for unfiltered J13 water after being stored for 190 days. 
Interestingly, the water sample retained in the 200 -liter barrel container had higher colloid concentration in all sizes after 190 days storage (Figure 24). Figure 25 shows the results of total colloid concentrations in these storage treatments. It appears that total colloids decreased in all long-term storage treatments using Teflon containers. However, total colloid concentration increased for the water sample retained in the 200 -liter barrel. These data demonstrate that colloid size and total concentration are affected by long-term storage ( 190 days); even the bottle was not opened during the period of storage. It should be noted that all of these experiments were conducted using J13 water that was initially transported in a 200-liter barrel.

From this task, it is concluded that sample storage may affect the stability of colloidal material. The sample should be analyzed for colloids soon after it is collected. The 200-liter plastic barrel is not an ideal container for collecting water samples for colloid analyses.

\section{Task III. Colloid Characterization of Groundwater Collected from ER-20-5, \#1 and \#3 Wells}

In the third task, we have characterized the colloid concentration and size distribution for groundwater samples collected freshly from ER-20-5 Wells \#1 and \#3. In this work, we have developed and followed the colloid sampling protocol to avoid colloid contamination.

Both unfiltered and 0.45- $\mu \mathrm{m}$ nylon-filtered ER-20-5 Well \#1 water samples were collected on April 30, 1998. Colloid size distribution and total colloid concentration were analyzed in the lab within 24 hours after samples were collected in the field. Some colloidal material accumulated on top of the $0.45-\mu \mathrm{m}$ nylon filter is visible as yellowish-colored material during the filtration process. Because the filter was obviously clogged, it is reasonable to believe that some colloids with sizes smaller than $200 \mathrm{~nm}$ were also trapped on top of the filter. It is not surprising that the colloid concentration in the filtered sample is almost two orders of magnitude lower than that in the unfiltered sample (Figure 26). The trapped colloid materials were further analyzed by electron microscope to study their elemental composition, particle shapes and morphology, and mineral components.

J13 Water Collected in 200-Liter Barrel

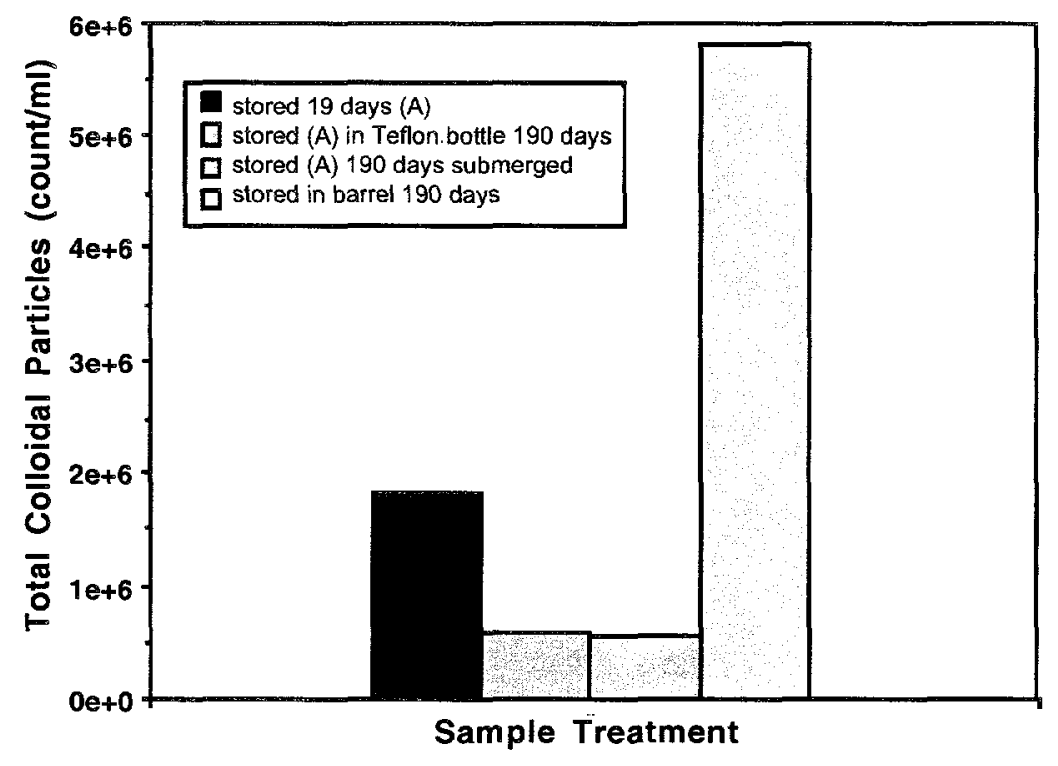

Figure 25. Total colloid concentration for $\mathbf{J} 13$ water before and after being stored for 190 days. 
Figures 26 and 27 show the colloid size distribution and total concentration for filtered and unfiltered ER-20-5 Well \#1 water samples. The total colloid concentration in unfiltered water samples is about $1 \mathrm{E} 10$ particles $/ \mathrm{mL}$. It is almost ten thousand times higher than the colloids concentration in J13 water. Colloid concentration in filtered samples is much lower than those in unfiltered samples. Obviously, some colloids, which are smaller than $0.45 \mu \mathrm{m}$ in size, did not pass through the filter. The particle size distribution results show that colloid concentrations continuously increase as the colloid particles get smaller. This result indicates that nanometer-sized colloids dominate in these groundwater samples.

Both scanning and TEMs were used to verify the size and shape of these nanometer-sized colloids. Colloidal materials that were trapped on top of the filter were collected for the study.

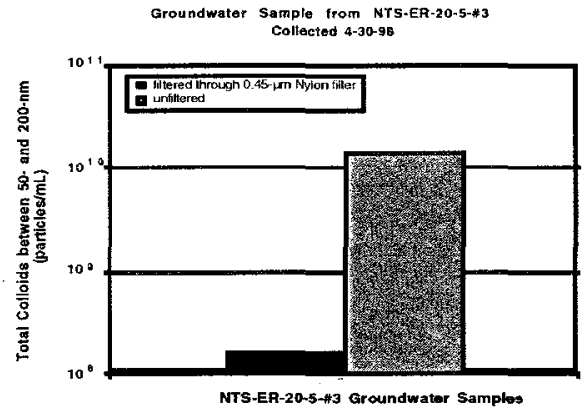

Figure 26. Total colloid concentration for the ER-20-5 Well \#1.

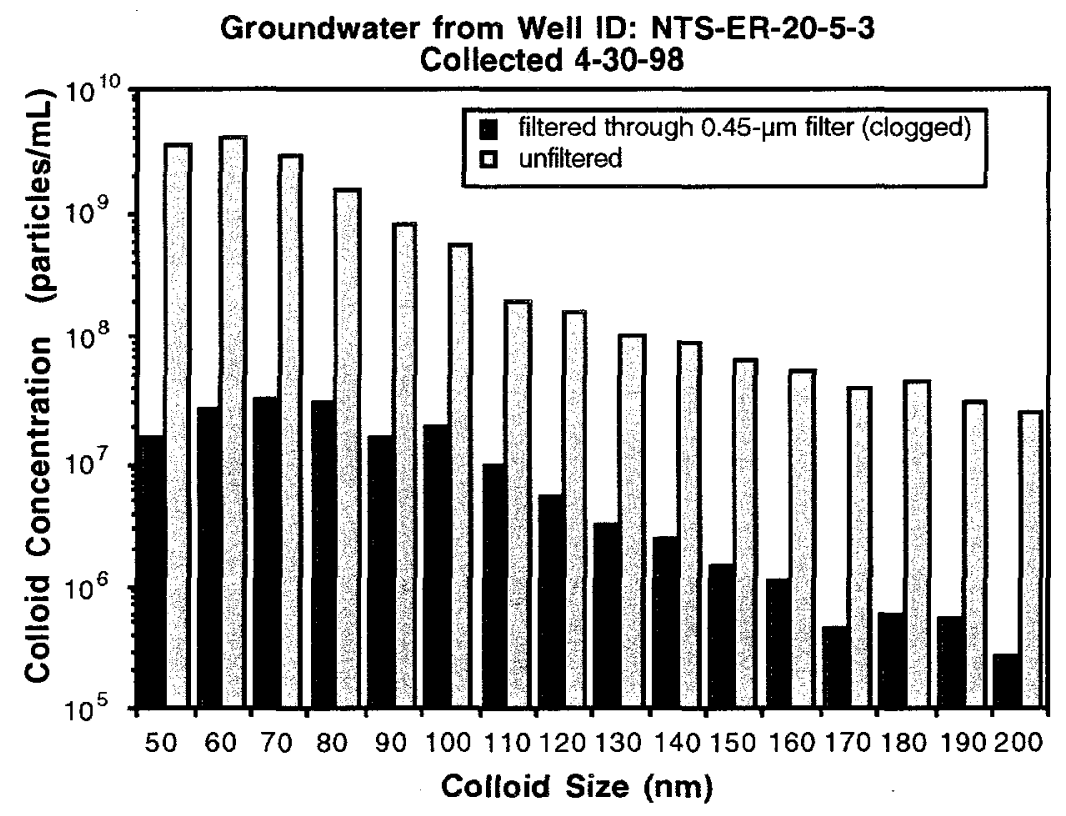

Figure 27. Colloid size distribution for the ER-20-5 Well \#1 water. 
The energy-dispersive spectrometer (EDS) from the scanning electron microscope shows the elemental composition of the colloidal material collected from the ER-20-5-1 Well. The EDS results (Figure 28) indicate that silicon and aluminum are dominant in the system. Small quantities of potassium, calcium, and iron are also present in the colloid material. The EDS spectrum (Figure 29) generated from the TEM shows a similar elemental composition to that in the scanning electron microscope (Figure 28) result. It should be noted that the $\mathrm{Cu}$ peaks around $8 \mathrm{keV}$ in Figure 29 are generated from the copper grid that was used as the TEM sample holder. These EDS spectra indicate that components normal for silicate minerals $(\mathrm{Al}, \mathrm{Si}, \mathrm{K}$, and $\mathrm{Ca}$ ) were present in the sample.

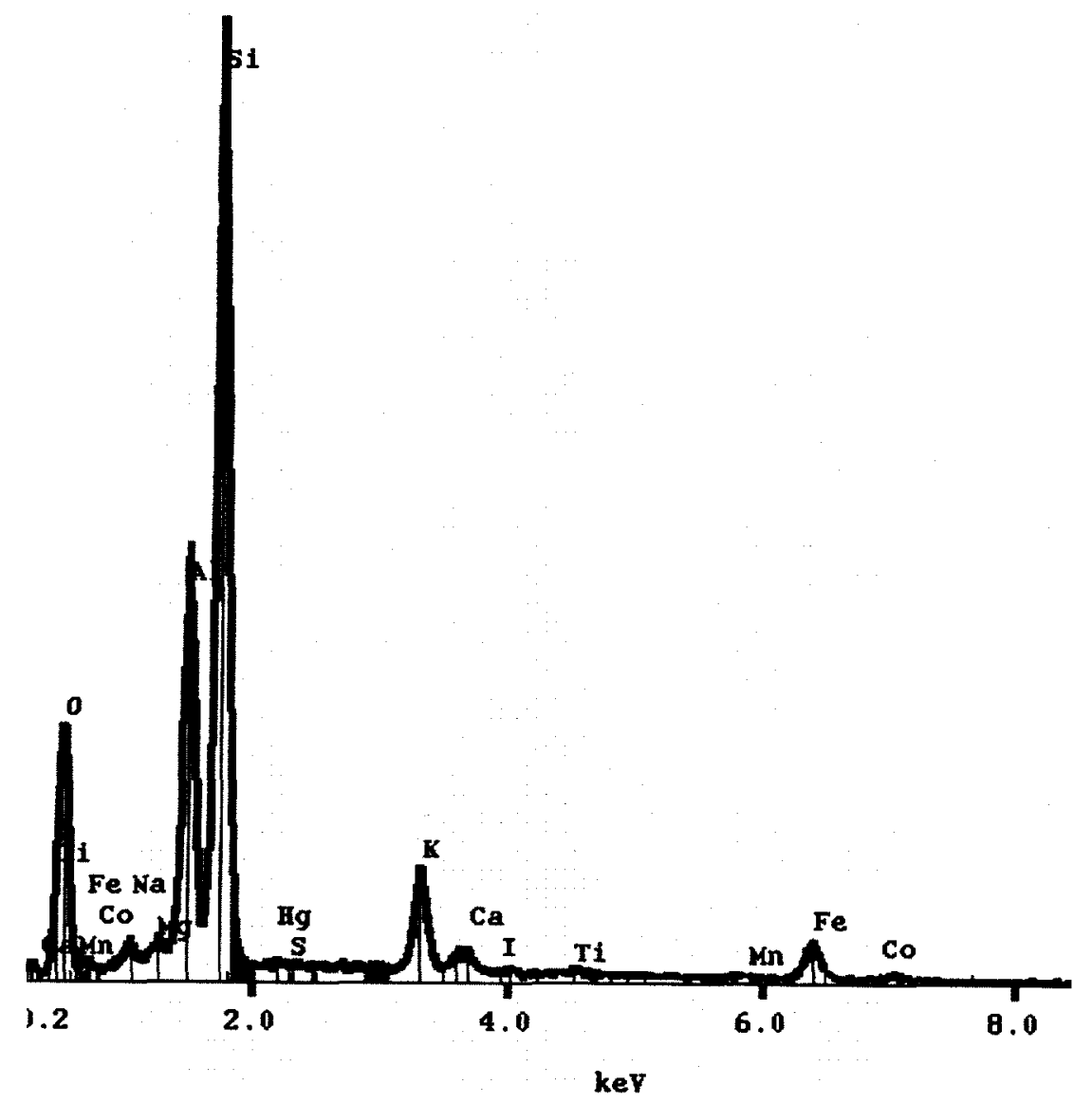

Figure 28. EDS results collected from the scanning electron microscope of colloids in ER-20-5 Well \#1 groundwater. 


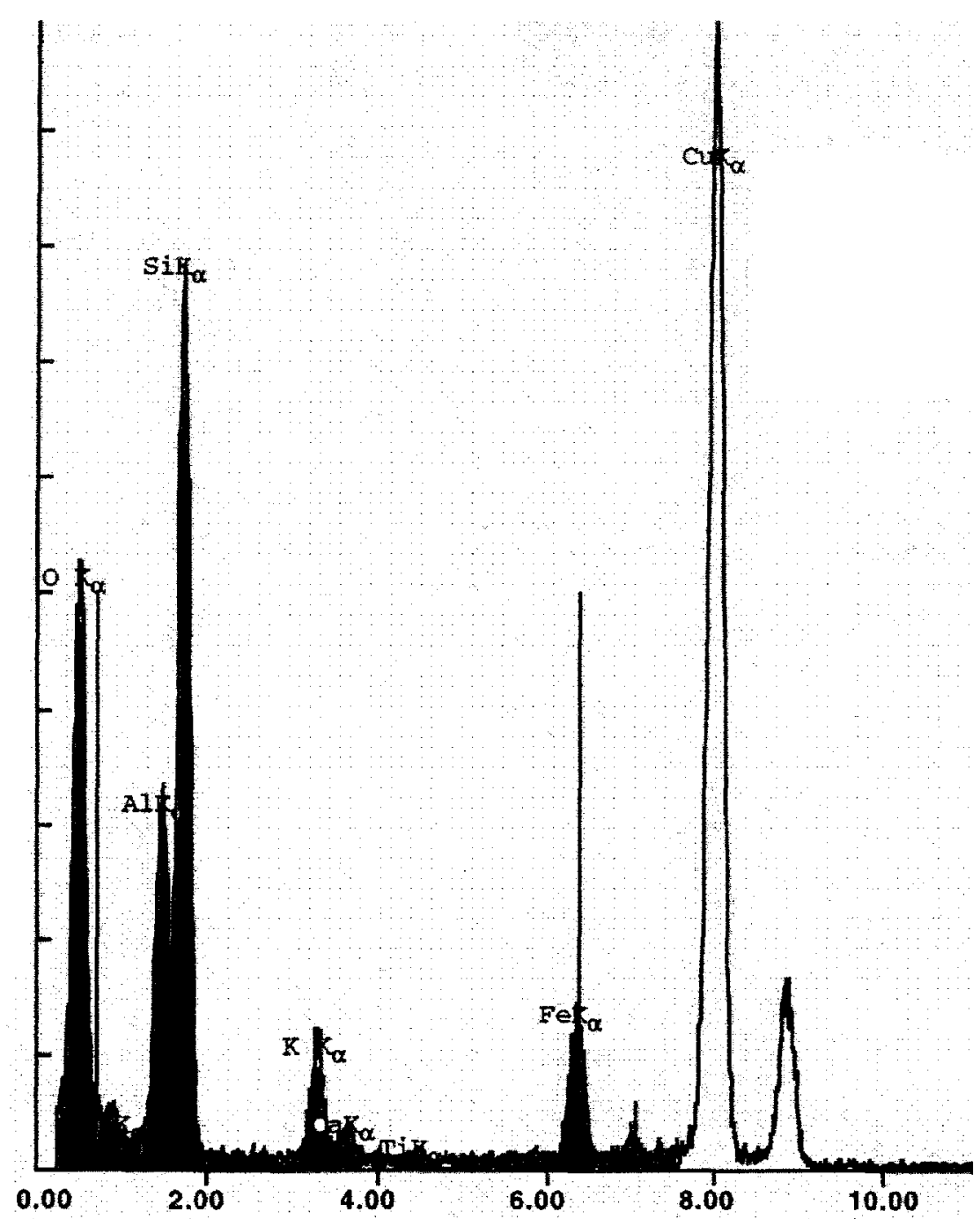

Figure 29. EDS results obtained from the TEM of colloidal material in ER-20-5 Well \#1 groundwater.

Because of the intrinsically small size of the colloidal material, the scanning electron microscope could not resolve the shape and size of each individual particle clearly. Micrographs from the SEM show stacks of micrometer-sized clusters. Therefore, the TEM was used to identify the colloidal shapes. Figures 30 to 32 show some electron micrographs generated from the Phillips CM-30 analytical TEM. These images clearly show the colloids with a variety of shapes and sizes. Results indicate that most colloids are less than $200 \mathrm{~nm}$ in size. It also suggests that colloidal particles have rigid edges even with sizes less than $200 \mathrm{~nm}$. Some particles seem to elongate in one dimension and to form rod shapes with one dimension as long as several hundred nanometers (Figures 32 and 33). 


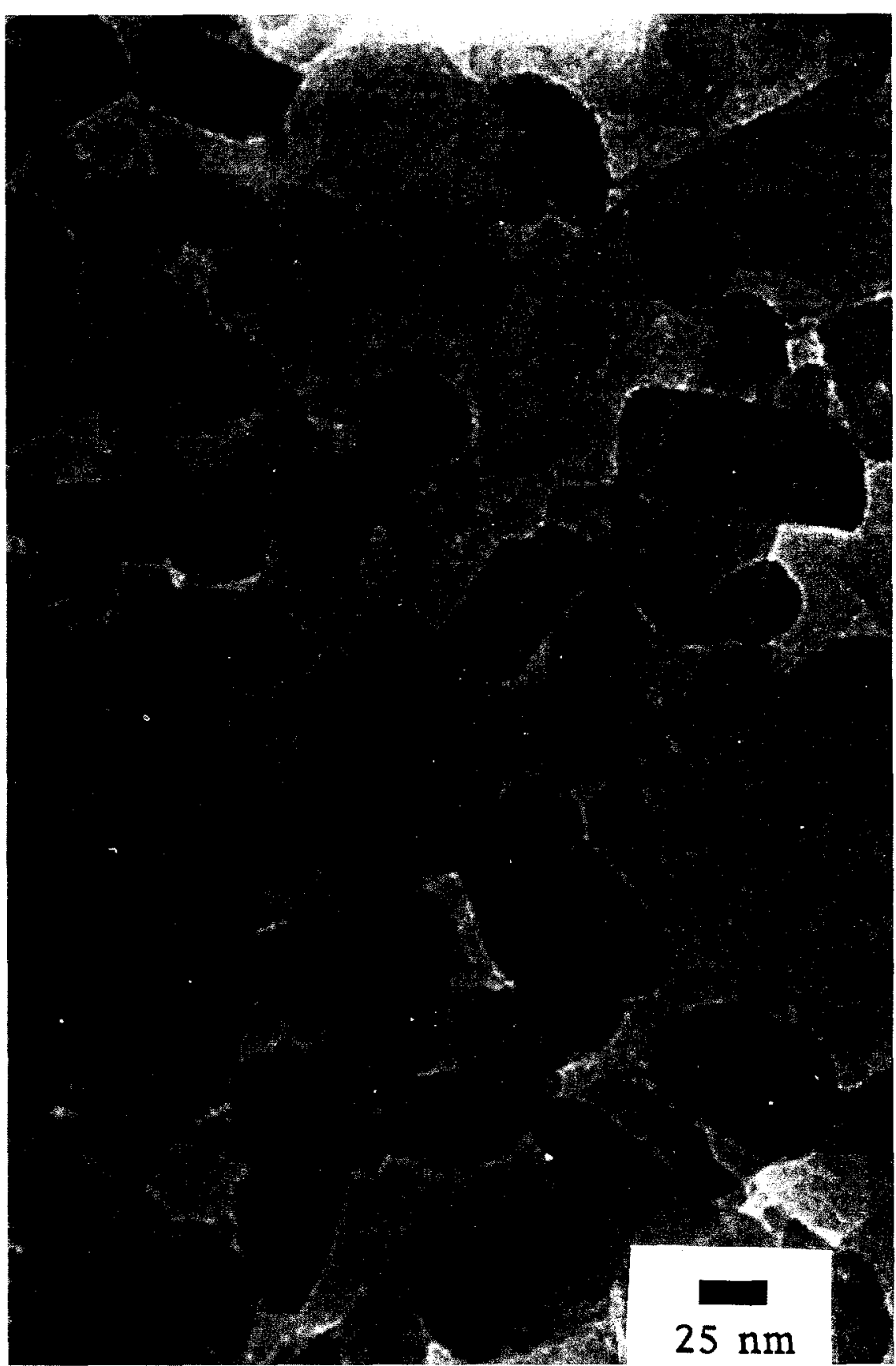

Figure 30. A transmission electron micrograph of colloidal particles collected from ER-20-5 Well \#1 groundwater. 


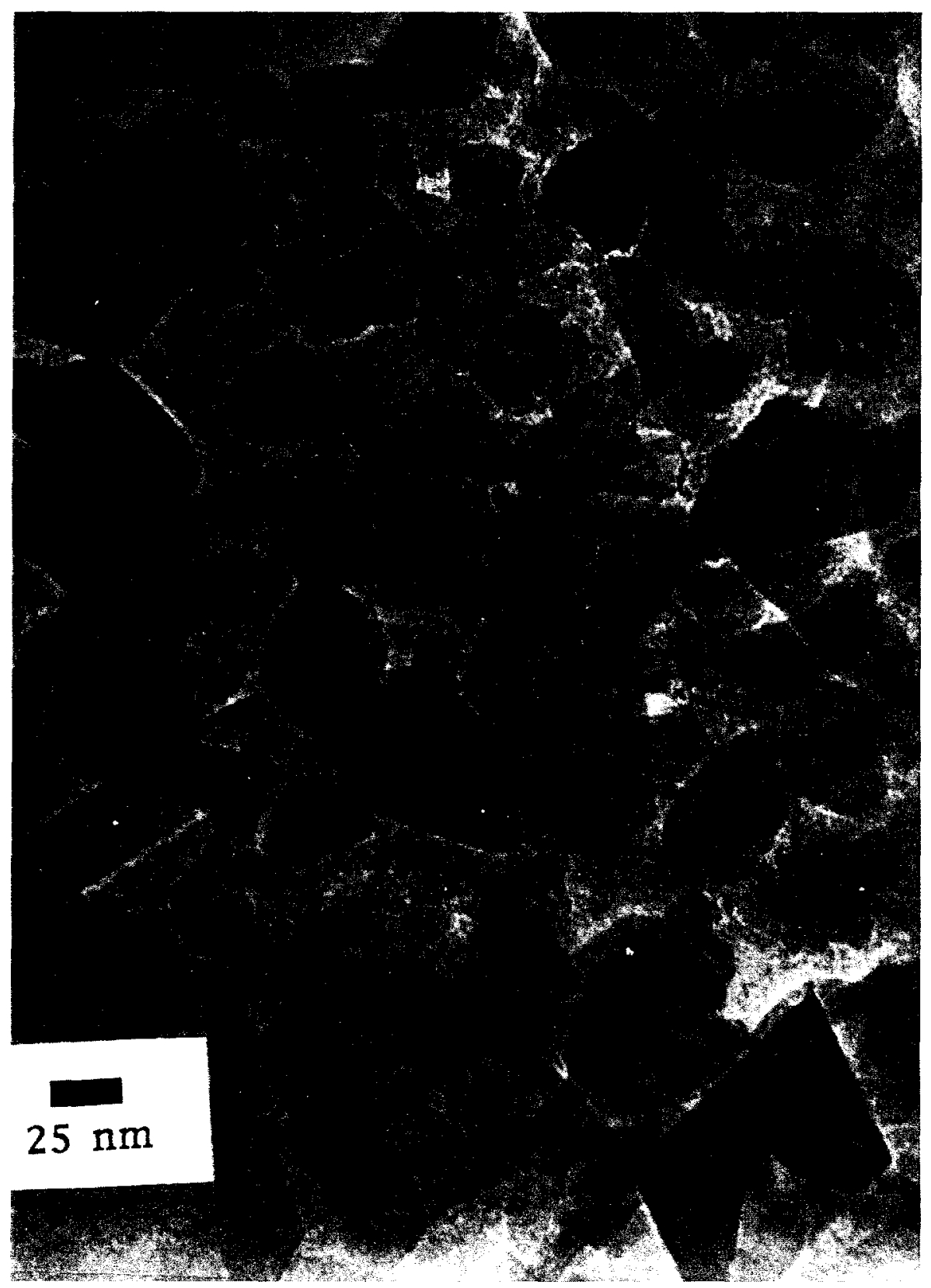

Figure 31. A transmission electron micrograph of colloidal particles collected from ER-20-5 Well \#1 groundwater. 


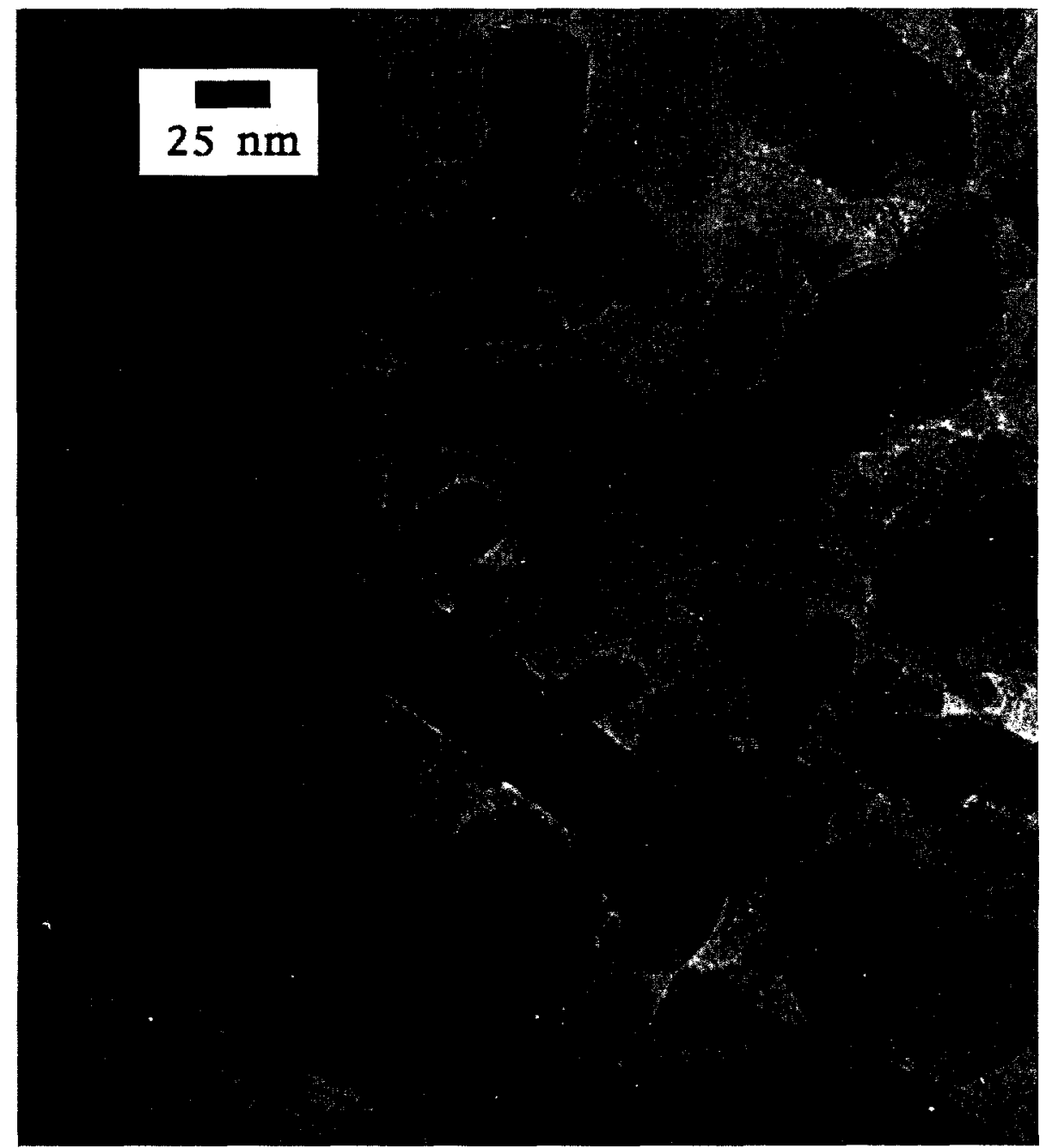

Figure 32. A transmission electron micrograph of colloidal particles collected from ER-20-5 Well \#1 groundwater. 


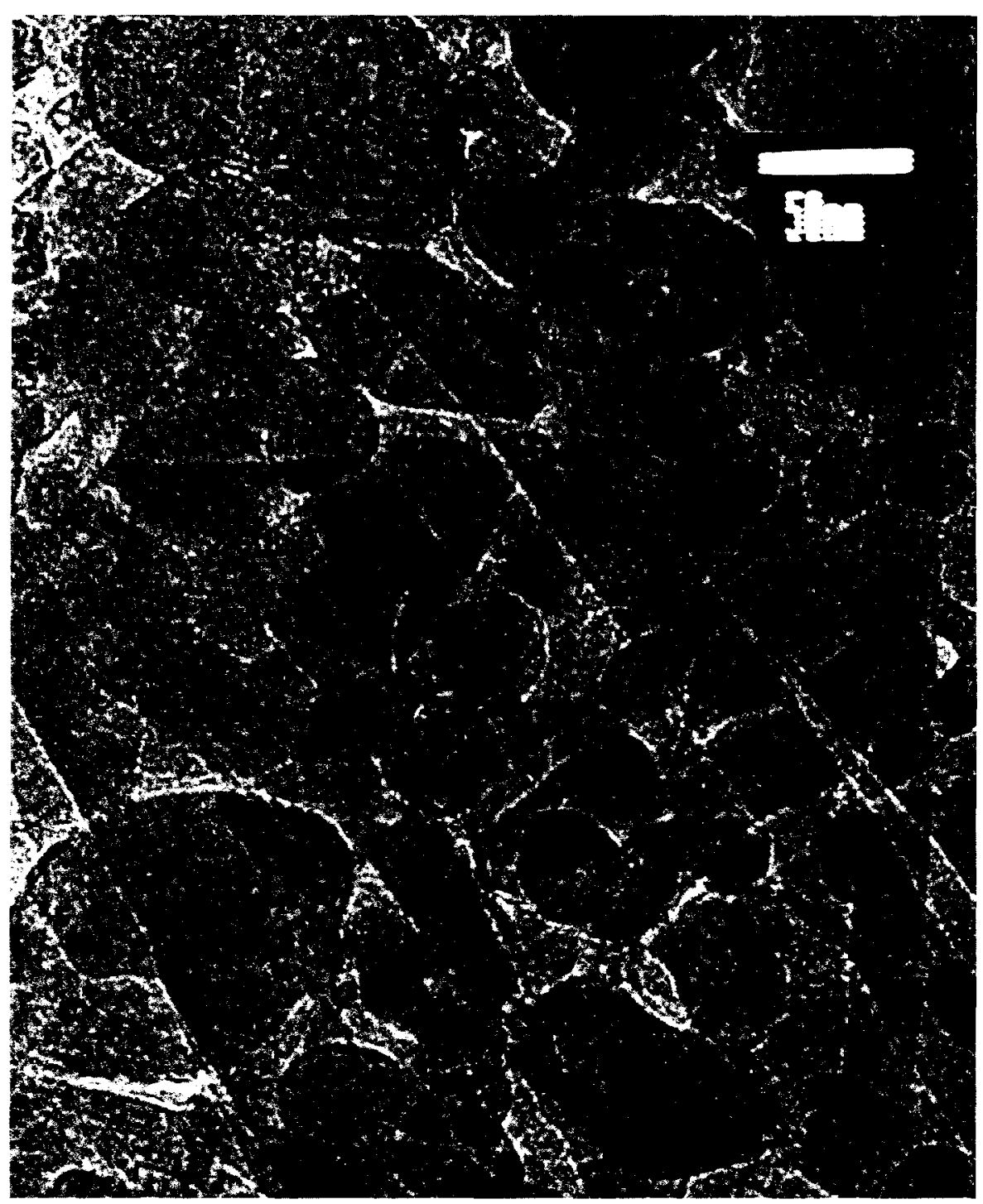

Figure 33. High-resolution transmission electron micrograph of colloidal particles collected from ER-20-5 Well \#1 groundwater. 
In addition to particle shapes and sizes, high-resolution TEM images (Figures 34 and 35) resolve the lattice fringe of individual colloidal particles. These images indicate that these nanometer-sized colloids are crystalline materials. Diffraction patterns from the TEM sample imply that smectites and silica minerals such as cristobalite and tridymite are present in the sample.

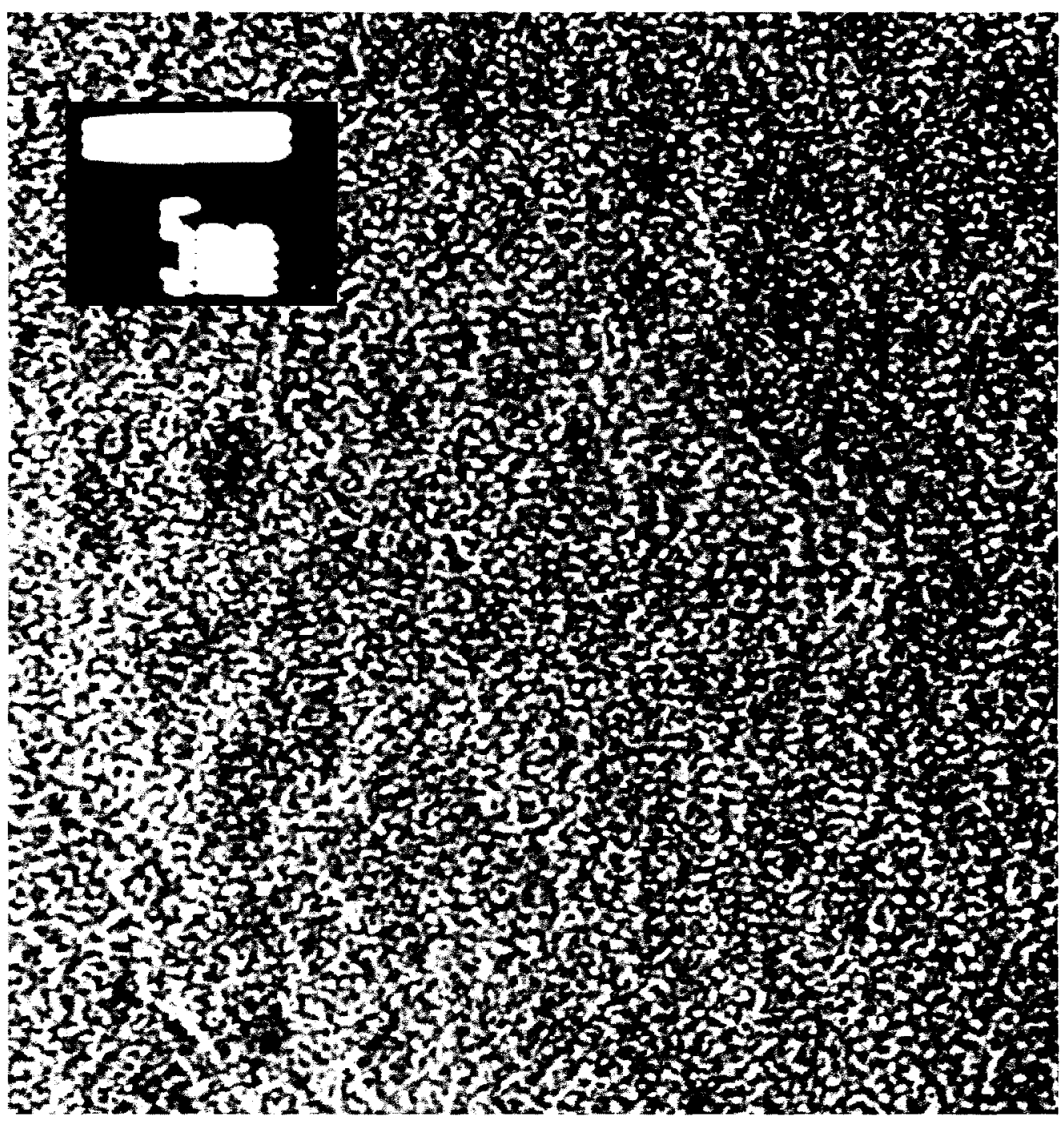

Figure 34. The transmission electron micrograph shows lattice fringe images of colloidal material collected from ER-20-5 Well \#1 groundwater. 


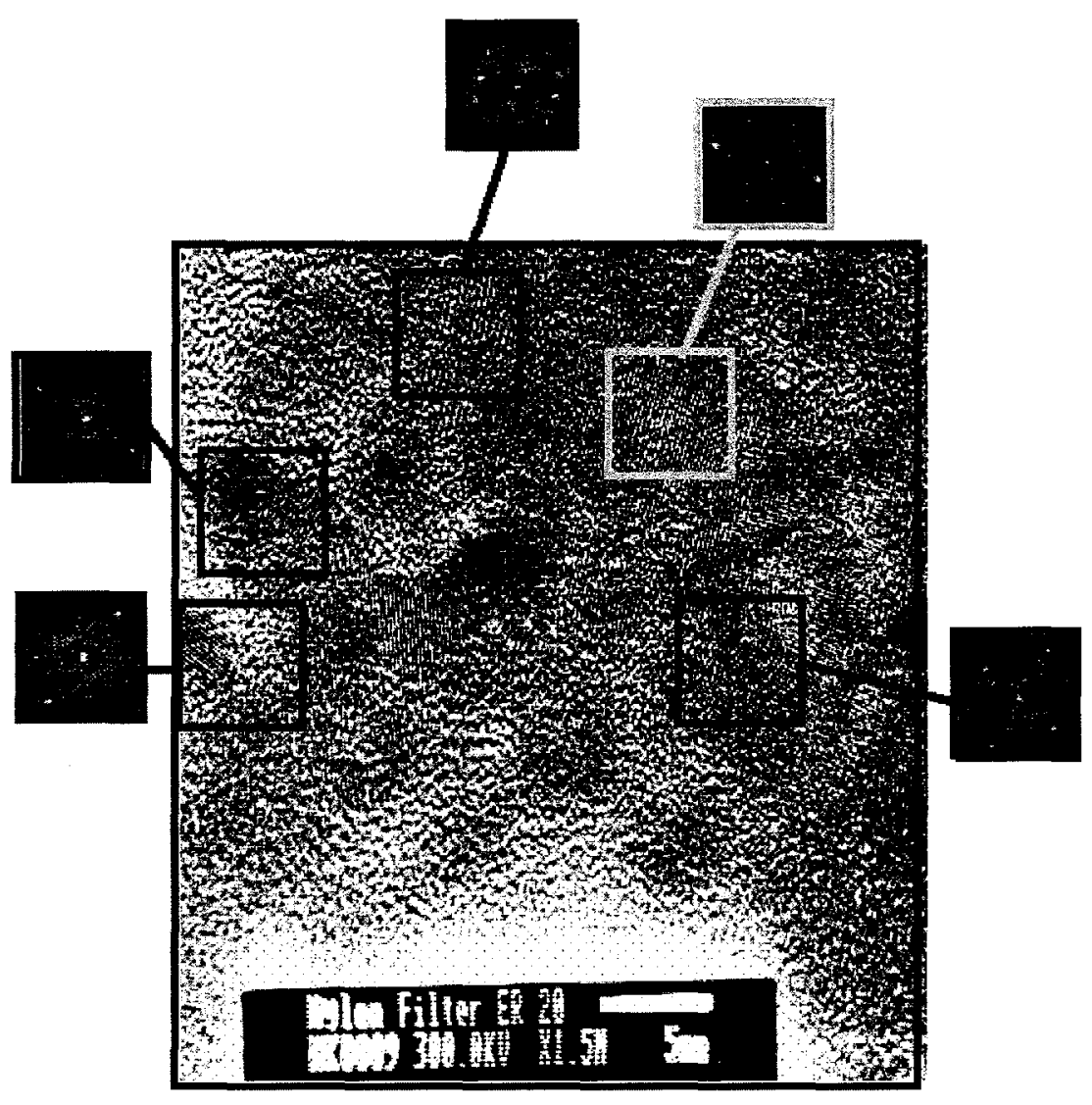

Figure 35. The high-resolution transmission electron micrograph shows lattice fringe images and computergenerated diffraction patterns of colloid material collected from ER-20-5 Well \#1 groundwater. 
A groundwater sample from the ER-20-5 \#1 Well was collected in the field by HRMP personnel on July 9, 1998. This sample was transported to Los Alamos and analyzed for colloids in the lab four days after it was collected (on July 13,1998). No filtration process was conducted because filters will trap colloids and affect the colloid size distribution and concentration.

The results of colloid size distribution and concentration for the ER-20-5 \#I Well sample are shown in Figures 36 and 37. For comparison, results of the ER-20-5 \#3 Well were also presented in these figures. Data indicate that the colloid concentrations and colloid size distributions presented in these two groundwater samples are similar. The measured total colloid concentrations between particle sizes in the 50- to 200-nm size range are 1.25E10 and 1.43E10 for the ER-20-5 \#1 Well and for the ER-20-5 \#3 Well, respectively.

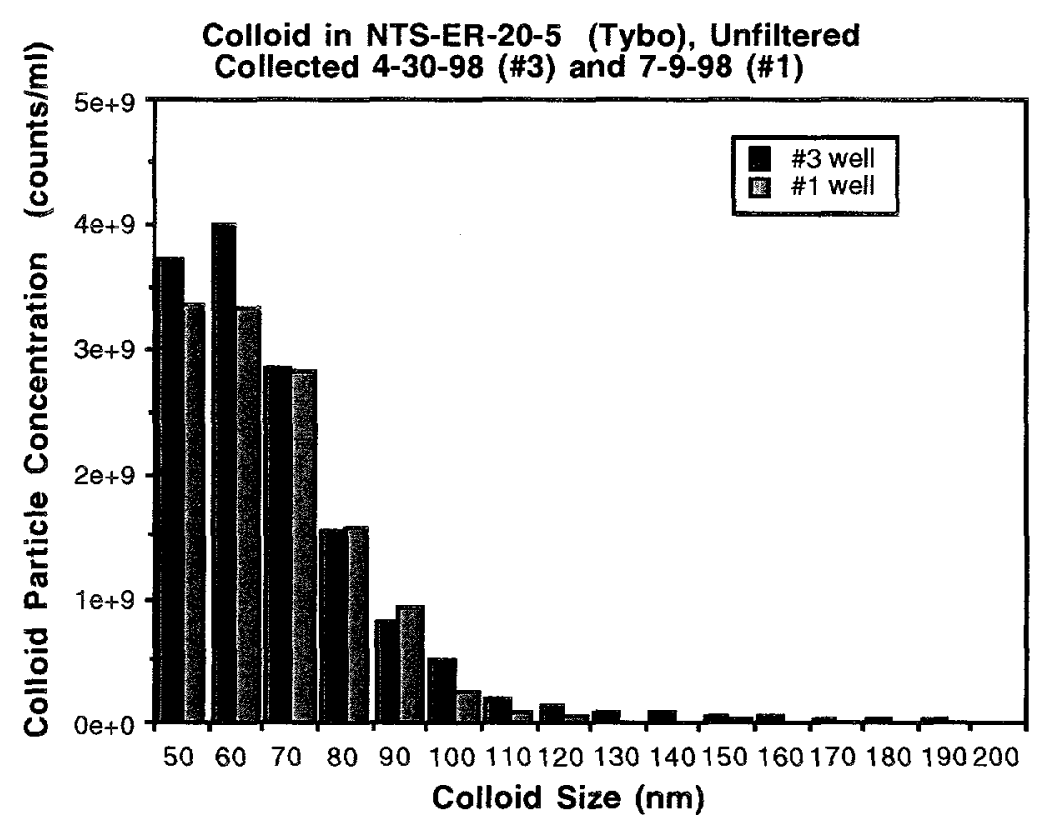

Figure 36. Colloid size distribution for the ER-20-5 Wells \#1 and \#3 samples. 


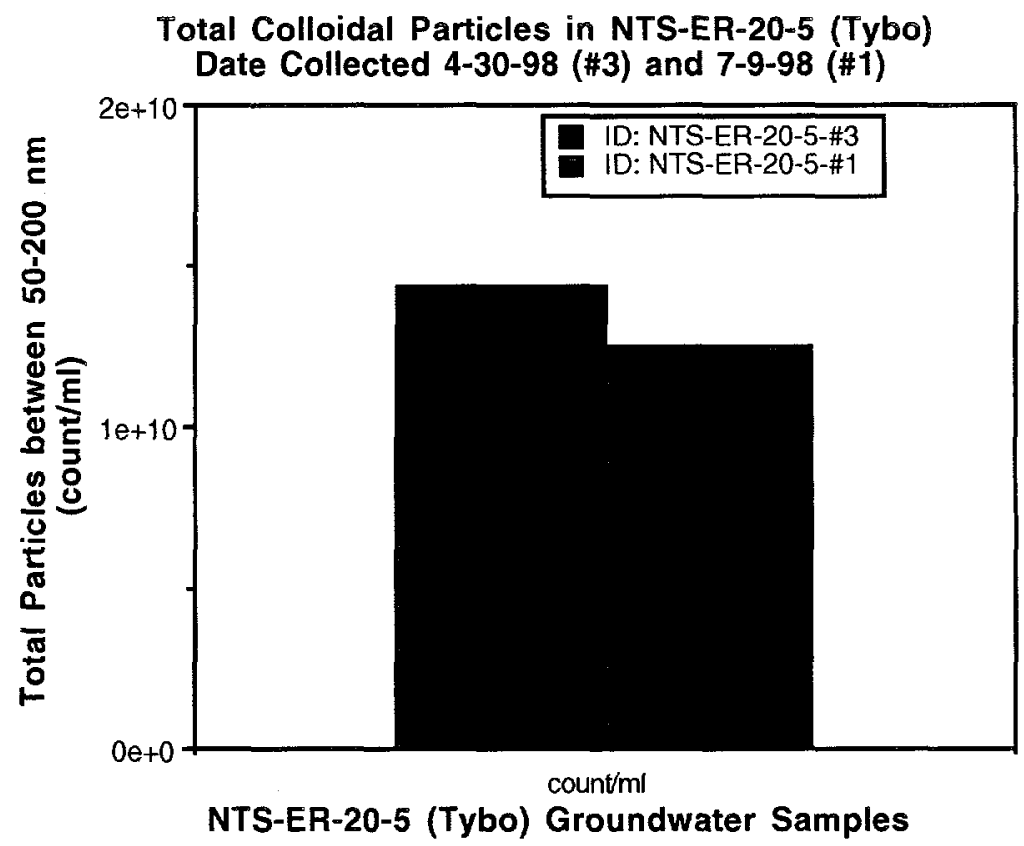

Figure 37. Total colloid concentration for ER-20-5 Wells \#1 and \#3.

For water collected from the ER-20-5 \#3 Well, there are slightly more colloids with particle sizes larger than $100 \mathrm{~nm}$ than those in the ER-20-5 \#1 Well, although less than 7\% of the total colloid in the \#3 Well are larger than $100 \mathrm{~nm}$ in size. For the ER-20-5 \#1 Well, less than $2 \%$ of the total colloids are larger than $100 \mathrm{~nm}$ in size. Within the 50 - to 100 $\mathrm{nm}$ colloidal particle size range, more colloids are present in water as the sizes of the colloids become smaller. These results suggest the nanometer-sized $(<100-\mathrm{nm})$ colloidal materials are possibly attributed to facilitated radionuclide transport in these groundwaters.

\section{Task IV. Colloid Analyses of Cheshire, U20n, Groundwater Samples}

In Task IV, we analyzed colloid concentration and size distribution in Cheshire (U20n ps1 ddh) water samples. Two sets of samples were obtained from HRMP field sampling personnel. The first set of samples was collected from the upper transmissive zone, (at around a well depth of 2,511 feet) on July 28, 1998. This sample was received and analyzed on July 30,1998 . Part of the sample was filtered through a $0.45-\mu \mathrm{m}$ nylon filter in the lab. In this filtration study, we used a small quantity of water (about $125 \mathrm{ml}$ ) to minimize clogging the filter. The results of colloidal concentration and size distribution for samples collected at a depth of 2,511 feet are reported in Figures 38 and 39. About $2 \mathrm{E} 8$ particles $/ \mathrm{mL}$ were found in these samples. The colloid size distributions for filtered and unfiltered samples are similar, although less colloids were present in the filtered sample. Apparently, some colloid particles that are smaller than the filter pore size had been trapped on top of the filter. It is obvious that filtration is not a reliable method for colloid separation. 


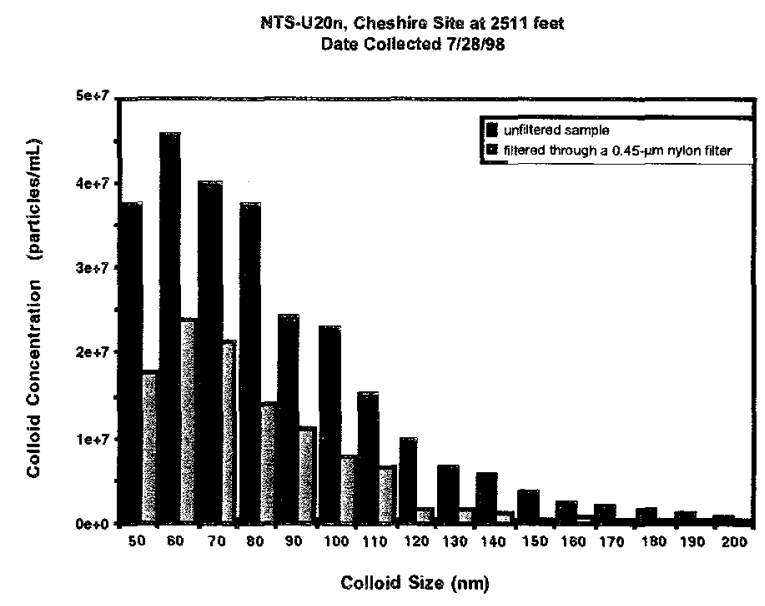

Figure 38. Unfiltered and $0.45-\mu \mathrm{m}$ filtered colloid size distribution in groundwater samples collected from Cheshire, U-20n.

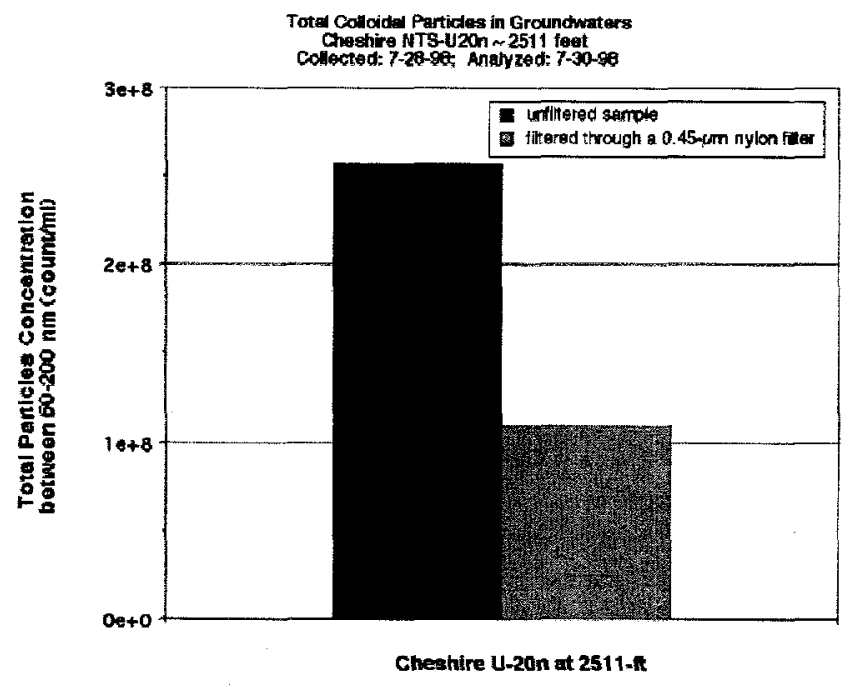

Figure 39. Unfiltered and $0.45-\mu \mathrm{m}$ filtered colloid concentration in groundwater samples collected from Cheshire, U-20n. 
A second set of samples from the Cheshire site was collected at a lower interval (around 4,200 $\mathrm{ft}$ in depth) on September 21, 1998, by HRPM personnel. This sample is labeled as PP\#1 dh and was analyzed for colloids on September 28,1998 . Although this zone had been pumped to remove materials associated with drilling, the water sample acquired a yellowish color.

The results from the Cheshire water samples are shown in Figures 38 to 41 . The colloidal size distributions from these two zones are rather similar, but the lower zone has about 20 times more colloids. This result is different from those from ER20-5 \#1 and \#3 wells, which show similar colloid concentrations, although water samples were collected from different depths. Colloid distribution results indicate that particles smaller than $100 \mathrm{~nm}$ dominate in the system.

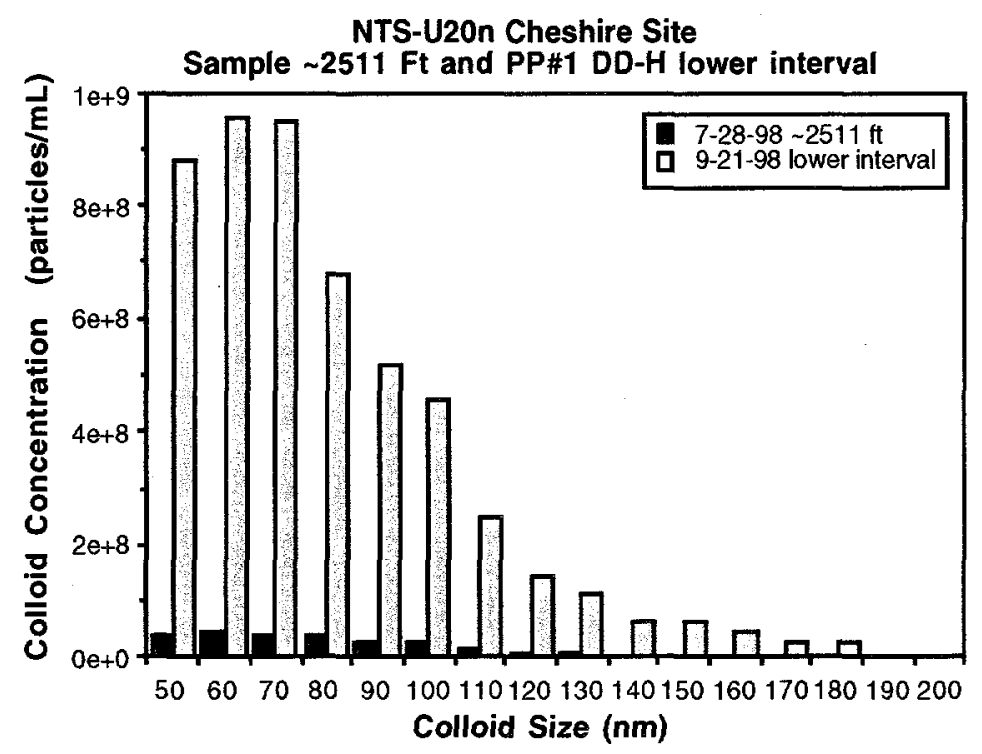

Figure 40. Colloid size distribution in groundwater samples collected from Cheshire, U-20n. 


\section{Total Colloidal Particles in Cheshire NTS-U20n}

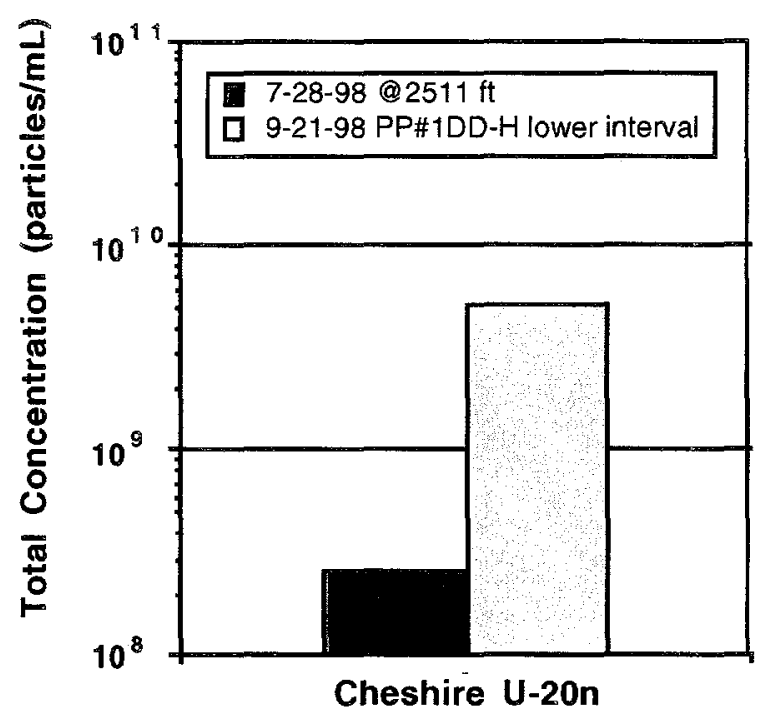

Figure 41. Total colloid concentration in groundwater samples collected from Cheshire, U-20n. 